\title{
The Bayesian Inversion Problem for Thermal Average Sampling of Quantum Systems
}

\author{
Ziheng Chen ${ }^{* 1}$ and Zhennan Zhou ${ }^{\dagger 2}$ \\ ${ }^{1}$ Department of Mathematics, The University of Texas at Austin, Austin 78705, USA \\ ${ }^{2}$ Beijing International Center of Mathematical Research, Peking University, Beijing 100871, P.R. China
}

February 21, 2020

\begin{abstract}
In this article, we propose a novel method for sampling potential functions based on noisy observation data of a finite number of observables in quantum canonical ensembles, which leads to the accurate sampling of a wide class of test observables. The method is based on the Bayesian inversion framework, which provides a platform for analyzing the posterior distribution and naturally leads to an efficient numerical sampling algorithm. We highlight that, the stability estimate is obtained by treating the potential functions as intermediate variables in the following way: the discrepancy between two sets of observation data of training observables can bound the distance between corresponding posterior distributions of potential functions, while the latter naturally leads to a bound of the discrepancies between corresponding thermal averages of test observables. Besides, the training observables can be more flexible than finite samples of the local density function, which are mostly used in previous researches. The method also applies to the multi-level quantum systems in the non-adiabatic regime. In addition, we provide extensive numerical tests to verify the accuracy and efficiency of the proposed algorithm.
\end{abstract}

\section{Introduction}

It has long been a challenging problem to establish inversion theories in quantum canonical ensembles (see Méhats and Pinaud, 2010, Lemm et al., 2000, Lemm, 2000, Lemm et al., 2001, Habeck, 2014, Lemm et al., 2005, Nguyen et al., 2017). One of the difficulties lies in the fact that the quantum canonical ensemble is not directly tractable as the classical case. In this article, the following thermal average of a given observable $\widehat{A}$ is of our interest:

$$
\langle\widehat{A}\rangle=\frac{1}{\mathcal{Z}} \operatorname{Tr}[\exp (-\beta \widehat{H}) \widehat{A}]
$$

*stokes615@utexas.edu

${ }^{\dagger}$ zhennan@bicmr.pku.edu.cn 
where $\widehat{H}=-\frac{1}{2 M} \Delta+V(q)$ is the quantum Hamiltonian, $q \in \mathbb{R}^{d}, M$ is the particle mass, $V: \mathbb{R}^{d} \rightarrow \mathbb{R}$ is the potential function, $\beta$ is referred to as the inverse temperature and $\mathcal{Z} \triangleq \operatorname{Tr}[\exp (-\beta \widehat{H})]$ is the partition function. In general, the physical observable $\widehat{A}$ corresponds to a self-adjoint operator, whereas for simplicity, we only consider observables as functions of the position variable in this work.

There have been some previous works regarding the existence of such a potential function that yields the local density function $n$ exactly as given. In [Méhats and Pinaud, 2010, the authors show that, in a 1-dimensional quantum system with periodic boundary conditions, any positive density function corresponds to a unique density operator minimizing the quantum free energy. Furthermore, the density operator is in the form of $\exp (-(-\Delta+V(x)))$ where $V$ is the chemical potential. The result is extended in Méhats and Pinaud, 2011 to a multi-dimensional case as well as unbounded domains and non-linear interactions in Hartree or Hartree-Fock systems. On the classical counterpart, Chayes et al., 1984 addresses the problem whether there exists an external potential corresponding to a given equilibrium single particle density. Results are established for both the canonical and grand canonical distributions. The Hamiltonian concerned is in the form of $H\left(x_{1}, x_{2}, \ldots x_{n}\right)=W\left(x_{1}, x_{2}, \ldots x_{n}\right)+\sum_{i=1}^{n} U\left(x_{i}\right)$ where $W$ is known (not required to be symmetric) and $U$ is unknown. Under a few integrable conditions, $U$ can be uniquely determined by local density function. A following work Navrotskaya, 2014 extends the conclusion to functions $U$ that may symmetrically contain more than one particle coordinate $x_{i}$.

Some other works lay emphasis on recovering the potential landscape numerically based on observations to the system. A first work Lemm et al., 2000 models the likelihood of potential functions based on position observables with finite given eigenvalues and the correspondingly training data $D$, which leads to an expression for the likelihood based on the wave function. Besides, the inversion process is done by maximizing a posteriori which involves taking the variational derivative on the posterior probability and the numerical implementation is based on the gradient descent algorithm. In Lemm, 2000, the effect of consecutive measurement at non-equilibrium is taken into account. Under the assumption of a time-independent Hamiltonian, the algorithm proposed in the previous work is adapted and amended by considering how the derivative of wave function depends on the time intervals between successive measurements. In Lemm et al., 2001, the authors discussed how to design priors to recover special properties in potential functions, such as periodicity (as for a distorted crystal surface) or expected discontinuities. A later work Lemm et al., 2005 rewrites the formula for the thermal average by using the Feynman path integral. To effectively (in numerical sense) evaluate the functional derivative of wave function, the stationary phase approximation is deployed.

The inverse problems in quantum statistics, similarly to those in other fields, can often be ill-posed or highly under-determined. At the same time, there is a wide gap between the works mentioned above: on one side, exact potential functions can be found in certain idealized scenarios, but only on a theoretical level; on the other side, some computational methods have been designed, but there is no clear conclusion on numerical convergence or stability. To merge the gaps, we adopt the Bayesian inversion framework to the quantum thermal average problem. In this work, a direct application on the inference of the potential functions is achieved by using this framework. However, due to the special structure of the quantum thermal average, i.e. the presence of the ring polymer potential, the Bayesian sampling process is much more challenging than its counterpart for classical systems. Thanks to the recent understanding of the continuum limit of the ring polymer representation, the Gibbs 
distribution on the infinite-dimensional configuration space serves as a crucial component in deriving efficient algorithms and proving stability results in spite of other technical difficulties. Compared to deterministic approaches for inverse problems, the Bayesian inversion method is more favorable for infinite-dimensional problems (see e.g. Stuart, 2010, Dashti and Stuart, 2017]). The advantage of this method is that it does not aim to identify the maximum point of the likelihood, but rather transforms the optimization problem into a sampling problem which naturally provides a statistical result for the inverse problem. There are a few following works applying this framework, for example, to the determination of the initial condition for Navier-Stokes equation in Cotter et al., 2010, to a three-dimensional global seismic wave propagation inverse problem with hundreds of thousands of parameters in Bui-Thanh et al., 2013 and to benchmarks in Higher-Order Ice Sheet Models in Petra et al., 2014.

The advantages of using this framework are mainly four-fold.

- Firstly, the Bayesian framework views the solution to the inverse problem as a posterior distribution that modifies the a priori knowledge of the unknown by assimilating the noisy observation data. It is impossible to uniquely pinpoint the potential function based on only a finite number of observations, whereas the Bayesian inversion framework admits multiple possible solutions with varying weights described by a posterior distribution. Based on Bayes's formula, the inversion from the training observations to the posterior distribution has a statistical sense. Furthermore, the stability of the result obtained from the inversion process is also ensured on a theoretical level.

- Secondly, the use of such framework also naturally leads to an efficient numerical algorithm. By building a Markov chain with the posterior distribution as the desired invariant distribution, the potential function can be sampled, providing further predictions on test observations. In the numerical section, we will show that the ground truth of test observations can be recovered if the noise covariance is assumed to be relatively small. This result confirms that not only the posterior distribution is stable with respect to noises, but also the predictions on test observations are practical and meaningful. Besides, the truncation error introduced in the ring polymer representation can be quantitatively estimated, which makes the ring polymer representation a superior asymptotic approximation compared with the static phase approximation adopted in Lemm et al., 2005.

- Moreover, the restrictions on training observables in this work can be largely relaxed, while in the previous works (see Lemm et al., 2000 and Lemm, 2000) the only viable option is the position observable confined in a small neighborhood of the most likely positions. In the following theorem statement, we will demonstrate that the training and testing observables can be expanded to bounded continuous functions. This greatly helps us to relax the strict requirements applied on practical physical measurements and ensure a wider application in real-life experiments.

- Last but not least, the Bayesian Inversion Framework used in this work also helps build a seamlessly connection between theory and algorithm in the infinite-parameter regime. There are also some other works focusing on problems that share a similar background with this inverse quantum thermal average problem. For example, the inverse Ising problem has been a popular 
topic. In Habeck, 2014, the authors adopt the Sequential Monte Carlo algorithm to infer the parameter $\lambda$ in systems with Hamiltonian $E(x)=\sum_{k=1}^{K} \lambda_{k} f_{k}(x)$. In Nguyen et al., 2017, the authors reviewed ways of recovering parameters in the Ising-type Hamiltonian $H(s)=$ $-\sum_{i} h_{i} s_{i}-\sum_{i<j} J_{i j} s_{i} s_{j}$. Methods mentioned in these works, however, only apply to a finiteparameter regime. As is emphasized in Stuart, 2010, avoiding discretization until the last possible moment and valuing the infinite-dimensional nature of the framework enables us to examine the coherence between theoretical results under the continuum limit and numerical implementation based on the ring polymer representation.

The outline of this paper is listed as follows. In Sec. 2, we review previous works addressing the sampling problem in quantum canonical ensembles. The inversion process theory is established in Sec. 3 . the numerical algorithm is proposed in Sec. 4 and a prior analysis is performed in Sec. 5 After that, we show a few numerical studies in Sec. 6.1 6.2 and 6.3 for systems of 1 and 2 levels correspondingly. The numerical tests also help us to verify the theoretical results proposed in Sec. 5 and gain further insights.

\section{Review on Quantum Thermal Average}

First, we summarize the mathematical model and the numerical approach of the forward problem, i.e. the thermal average of an observable in a quantum system. In the following, we introduce the ring polymer representation of the thermal average, its continuum limit and the path integral molecular dynamics method for numerical simulation.

\subsection{Ring polymer representation}

The ring polymer representation, first proposed in Feynman, 1965 (Sec. 10) and widely used in chemical physics (e.g. see Sec. 2.9 in Kleinert, 2009), approximates the thermal average of observable $\widehat{A}$ with respect to the classical Gibbs distribution in the $d N$-dimensional space for ring polymer $\boldsymbol{q}=\left(q_{1}, \ldots, q_{N}\right) \in \mathbb{R}^{d N}, q_{i} \in \mathbb{R}^{d}$ as

$$
\langle\widehat{A}\rangle=\frac{1}{\mathcal{Z}_{N}} \int_{\mathbb{R}^{d N}}\left[\frac{1}{N} \sum_{i=1}^{N} A\left(q_{i}\right)\right] e^{-S_{N}(\boldsymbol{q})} d \boldsymbol{q}+\mathcal{O}\left(N^{-2}\right),
$$

where the action, depending on the potential function $V: \mathbb{R}^{d} \rightarrow \mathbb{R}$, is given by

$$
S_{N}(\boldsymbol{q}) \triangleq \beta_{N} \sum_{i=1}^{N}\left[\frac{M\left|q_{i}-q_{i+1}\right|^{2}}{2 \beta_{N}^{2}}+V\left(q_{i}\right)\right]
$$

and $\mathcal{Z}_{N} \triangleq \int_{\mathbb{R}^{N}} e^{-S_{N}(q)} d q$ is the normalization constant and $\beta_{N} \triangleq \beta / N$.

Define the shorthand notation $\bar{A}(\boldsymbol{q}) \triangleq \frac{1}{N} \sum_{i=1}^{N} A\left(q_{i}\right)$. Eqn. 1 indicates that the thermal average $\langle\widehat{A}\rangle$ can be approximated by the expectation of $\bar{A}(\boldsymbol{q})$ with respect to the following Gibbs distribution

$$
\pi_{N}^{V}(d \boldsymbol{q}) \triangleq \frac{1}{\mathcal{Z}_{N}} e^{-S_{N}(\boldsymbol{q})} d \boldsymbol{q}
$$


However, since the dimension of the configuration space is $d N$, a direct numerical integration based on $\pi_{N}^{V}$ is too expensive due to large $N$ required by reducing the approximation error. To avoid the curse of dimensionality, a few numerical sampling methods are designed to approximate the integration by averaging a time series based on Eqn. 3, we will further discuss this topic in Sec. 2.3 .

\subsection{Continuum limit}

As the division number $N$ approaches infinity, there is a formal limit for the ring polymer configuration $\boldsymbol{q}$ as well for the action $S_{N}$. For given bead number $N$, a piece-wise linear path $\mathfrak{q}_{N}$ can be constructed by setting $\mathfrak{q}_{N}\left(j \beta_{N}\right)=q_{j}$ and periodic on $[0, \beta]$, so the formal limit of $\mathfrak{q}_{N}$ as $N \rightarrow \infty$ is also in the space $\mathcal{L} \mathbb{R}^{d} \triangleq\left\{\mathfrak{q}:[0, \beta] \rightarrow \mathbb{R}^{d}, \mathfrak{q}(0)=\mathfrak{q}(\beta)\right\}$. The corresponding limit for the action is given by

$$
S(\mathfrak{q}) \triangleq \int_{0}^{\beta}\left[\frac{M}{2}|\dot{\mathfrak{q}}|^{2}+V(\mathfrak{q}(\tau))\right] d \tau
$$

so the thermal average, taking the limit $N \rightarrow \infty$, can be formally written as

$$
\langle\widehat{A}\rangle=\frac{1}{\mathcal{Z}} \int_{\mathcal{L}_{\mathbb{R}^{d}}}\left[\frac{1}{\beta} \int_{0}^{\beta} A(\mathfrak{q}(\tau)) d \tau\right] \exp (-S(\mathfrak{q})) D[\mathfrak{q}]
$$

where $D[\mathfrak{q}]$ denotes integration over all paths $\mathfrak{q}$ with $\mathfrak{q}(0)=\mathfrak{q}(\beta)$ and $\mathcal{Z} \triangleq \int_{\mathcal{L} \mathbb{R}^{d}} e^{-S(q)} D[\mathfrak{q}]$ is the normalization constant.

Eqn. 4 allows us to define the following formal Gibbs distribution (also see [Feynman, 1965] Sec.10 and [Lu and Zhou, 2018b]) on the configuration space

$$
\pi^{V}(d \mathfrak{q}) \triangleq \frac{1}{\mathcal{Z}} \exp (-S(\mathfrak{q})) D[\mathfrak{q}]
$$

so that the thermal average in Eq. 4 can be rewritten as

$$
\langle\widehat{A}\rangle=\mathbb{E}_{\pi^{V}(d \mathfrak{q})}\left[\frac{1}{\beta} \int_{0}^{\beta} A(\mathfrak{q}(\tau)) d \tau\right]=\mathbb{E}_{\pi^{V}(d \mathfrak{q})} \bar{A}[\mathfrak{q}]
$$

where the shorthand notation is defined as

$$
\bar{A}[\mathfrak{q}] \triangleq \frac{1}{\beta} \int_{0}^{\beta} A(\mathfrak{q}(\tau)) d \tau .
$$

Note. To emphasize the dependency of $\langle\widehat{A}\rangle$ on the potential $V$, we use the notation $G^{A}(V)$ to represent the mapping from $V$ to the thermal average $\langle\widehat{A}\rangle$ where needed. On the corresponding part, the notation $G_{N}^{A}(V)$ is used to represent the mapping to the thermal average under the ring polymer representation, where $N$ is the number of beads on the ring $\boldsymbol{q}$. 


\subsection{Under-damped Langevin sampling}

To enhance the efficiency of the sampling process, an auxiliary momentum variable $\boldsymbol{p} \in \mathbb{R}^{d N}$ with artificial mass $M$ can be introduced Liu et al., 2016 [Zhang et al., 2017. In the augmented state space of position and momentum of ring polymer beads, the thermal average is given by

$$
\langle\widehat{A}\rangle=\frac{1}{\mathcal{Z}_{N}^{\prime}} \int_{\mathbb{R}^{N}} \int_{\mathbb{R}^{N}}\left[\frac{1}{N} \sum_{i=1}^{N} A\left(q_{i}\right)\right] e^{-\beta_{N} H_{N}(\boldsymbol{q}, \boldsymbol{p})} d \boldsymbol{q} d \boldsymbol{p}+\mathcal{O}\left(N^{-2}\right)
$$

where the Hamiltonian is given by

$$
H_{N}(\boldsymbol{q}, \boldsymbol{p})=\frac{1}{2 M}|\boldsymbol{p}|^{2}+\sum_{i=1}^{N}\left[\frac{M\left|q_{i}-q_{i+1}\right|^{2}}{2 \beta_{N}^{2}}+V\left(q_{i}\right)\right] .
$$

Therefore the classical Gibbs distribution in the extended phase space can be sampled by evolving the following dynamic system:

$$
\left\{\begin{array}{l}
d \boldsymbol{q}=\nabla_{\boldsymbol{p}} H_{N} d t \\
d \boldsymbol{p}=-\nabla_{\boldsymbol{q}} H_{N} d t-\gamma \boldsymbol{p} d t+\sqrt{\frac{2 \gamma M}{\beta_{N}}} d \boldsymbol{B} .
\end{array}\right.
$$

To numerically integrate the SODE in Eqn. 9, we use the BAOAB method which is proposed in Leimkuhler and Matthews, 2013a, further tested and compared against other variants (ABOBA and OBABO) in Leimkuhler and Matthews, 2013b and Liu et al., 2016, and investigated elaborately and shown useful for other types of thermostats in [Zhang et al., 2017].

\subsection{Multi-level system}

The thermal average of a given observable in a multi-level system has been explored in the previous works. In Menzeleev et al., 2014], kinetically-constrained RPMD is proposed to directly simulate electronically non-adiabatic chemical processes. In Ananth, 2013, the authors proposed mappingvariable RPMD which constructs continuous Cartesian variables for both electronic states and nuclear degrees of freedom; see also the review paper [Stock and Thoss, 2005].

Recently, a few works focus on the exact computation of multi-level systems. In Liu and Liu, 2018, three splitting schemes for the Boltzmann operator in the non-adiabatic representation are discussed in detail and multi-electronic-state PIMD is derived afterwards. In [Tao et al., 2018, a corresponding isomorphic Hamiltonian is introduced to fully recover the exact quantum Boltzmann distribution under the Boltzmann sampling with classical nuclear degrees of freedom. In this paper, we implement the method proposed by Lu and Zhou, 2017 where the non-adiabatic effect is added into consideration by modeling the surface hopping procedure as a Q-process. A following work Lu and Zhou, 2018a improves this method by introducing a multiscale integrator for the infinite swapping limit. We will supply the essential formula in Sec. B in the Appendix. 


\section{Inversion Process Based on Thermal Averages}

Sec. 2 has established the forward problem, i.e. given observable $\widehat{A}$ and potential $V$, the thermal average can be formulated as

$$
\boldsymbol{y}_{\text {truth }}=G^{A}(V) \text {. }
$$

However, an observation $\boldsymbol{y}$ from the real world or experiment may deviate from the ground truth $G^{A}(V)$ because of noise, possibly due to instrumental bias, measurement error, and thermal fluctuation. A simplest model for the noise $\boldsymbol{\eta}$ is the additive assumption, i.e.

$$
\boldsymbol{y}=G^{A}(V)+\boldsymbol{\eta},
$$

and the noise $\boldsymbol{\eta}$ is assumed independent of the potential function $V$. Since $\boldsymbol{\eta}$ is a random variable, a determinate result for the "best" $V$ barely makes sense. Nevertheless, it is sensible to presume a posterior distribution $\mu^{\boldsymbol{y}}(d V)$ of $V$ based on the noisy observation $\boldsymbol{y}$, given a known prior distribution on the space of all admissible potential functions. As the observable $\widehat{A}$ is used to recover the posterior distribution, we will name it as the "training observable". On the other hand, we in practice are interested in the inference of other thermal averages within the same ensemble, which can be viewed as a weak version of the inverse problem in quantum statistics. Such concern can be generalized to the thermal average of another observable $\widehat{O}$, named as the "testing observable".

Although the prediction from $\boldsymbol{y}$ to $\langle\widehat{O}\rangle$ may look like a classical statistics learning problem, the difficulty lies in the nonlinear nature of density operators with respect to the potential function and the observation data in quantum canonical ensembles. To address this both theoretically and numerically, we replace the inverse mapping with the posterior distribution $\mu^{\boldsymbol{y}}(d V)$. Instead of directly computing $G^{O}\left(V_{p}\right)$ by the point estimator $V_{p}$ (for example MAP), we treat $V$ as an intermediate random variable and thus shift our attention to the weighted average $\mathbb{E}_{\mu^{y}(d V)} G^{O}(V)$, where the weights are obtained by sampling the posterior distribution $\mu^{\boldsymbol{y}}(d V)$ induced by the noisy training observation. In fact, the

weighted-average $\mathbb{E}_{\mu^{y}(d V)} G^{O}(V)$ sampled by the algorithm can be written into $\mathbb{E}_{\pi^{y}(d \mathfrak{q})} \bar{O}[\mathfrak{q}]$, thus it suffices to study the property of the posterior distribution $\pi^{\boldsymbol{y}}(d \mathfrak{q})$ of the conditional variable $\mathfrak{q} \mid \boldsymbol{y}$.

The methodology in this work contains a direct application on the Bayesian inversion framework Dashti and Stuart, 2017 as well as some special considerations with regard to the structure of quantum thermal averages. The inversion procedure from observations $\boldsymbol{y}$ to potential functions $V$ is just a reformulation of how the posterior distribution is derived in such framework. Nevertheless, the posterior distribution on $V$ helps us to establish the posterior distribution on configurations $\mathfrak{q}$ and the weight average $\mathbb{E}_{\pi^{y}(d \mathfrak{q})} \bar{O}[\mathfrak{q}]$ of test observables $O$. It also helps in the following two aspects. The conclusion on stability is established by combining the theorem on stability in this framework and the fact that the Gibbs distribution continuously depends on potential $V$. The consistency of the numerical algorithm results from the equality of two thermal averages that are obtained from different perspectives.

In the following sections, we discuss the following properties of the posterior distribution:

Existence We formulate the inverse problem in this section. The posterior distribution $\mu^{\boldsymbol{y}}(d V)$ can be obtained from the prior distribution $\mu_{0}(d V)$ by evaluating the negative log potential $\Phi(V ; \boldsymbol{y})$ with the training observation $\boldsymbol{y}$. To further describe the conditional variable $\mathfrak{q} \mid \boldsymbol{y}$, we 
can view $V$ as an intermediate variable to obtain the posterior distribution $\pi^{\boldsymbol{y}}(d \mathfrak{q})$ based on the two conditional variables $V \mid \boldsymbol{y}$ and $\mathfrak{q} \mid V$. By the end $\mathbb{E}_{\mu^{y}(d V)} G^{O}(V)=\mathbb{E}_{\pi^{y}(d \mathfrak{q})} \bar{O}[\mathfrak{q}]$ is proved to confirm the consistency between our model and algorithm.

Solvability We demonstrate how to numerically sample the posterior distribution $\mu^{\boldsymbol{y}}(d V)$ and discuss some details in implementation in Sec. 4. The algorithm is an iterative procedure in a proposaldecision approach: in each iteration, a new potential proposal $\widehat{V}^{(k+1)}$ is drawn based on the previous sample $V^{(k)}$ and the acceptance probability is based on the comparison between current and previous observation errors. To be more specific, the algorithm can be abstracted as:

Initialization Obtain the ground truth observation $\boldsymbol{y}^{*}$. Draw $\widehat{V}^{(0)}=V^{(0)} \sim \mu_{0}(d V)$.

Proposal Draw $\widehat{V}^{(k+1)}$ based on $V^{(k)}$. Compute training observation $\widehat{\boldsymbol{y}}^{(k+1)}=G^{A}\left(\widehat{V}^{(k+1)}\right)$.

Decision With probability $a\left(\widehat{\boldsymbol{y}}^{(k+1)}, \boldsymbol{y}^{(k)} ; \boldsymbol{y}^{*}\right)$ which is consistent with the proposal scheme, accept, otherwise reject the proposal.

Accept $V^{(k+1)}=\widehat{V}^{(k+1)}$ and $\boldsymbol{y}^{(k+1)}=\widehat{\boldsymbol{y}}^{(k+1)}$.

Reject $V^{(k+1)}=V^{(k)}$ and $\boldsymbol{y}^{(k+1)}=\boldsymbol{y}^{(k)}$.

Stability We give some stability analysis in Sec. 5. We construct a series of proofs to show that the posterior distributions are stable under the disturbance of the noisy observation data.

The formulation of the inverse problem and the stability result will be discussed under the continuum limit, while the numerical scheme are based on the ring polymer representation in finite dimensions. Also, we assume the technical condition that the posterior distribution mentioned in the paper is absolutely continuous to the corresponding prior distribution (e.g. $\mathfrak{q} \mid V$ to $\mathfrak{q}, V \mid \boldsymbol{y}$ to $V$ ).

\subsection{Notations}

Without loss of generality, we assume that the physical space of the quantum particle is one-dimensional, i.e. $d=1$.

The space of $\beta$-periodic loops $\mathfrak{q}$ (in the continuum limit sense) is defined as

$$
X \triangleq \mathcal{L} \mathbb{R} \triangleq\{\mathfrak{q}:[0, \beta] \rightarrow \mathbb{R}, \mathfrak{q}(0)=\mathfrak{q}(\beta)\}
$$

while the space of $N$-bead ring polymer $\boldsymbol{q}$ is defined as

$$
X_{N} \triangleq \mathbb{R}^{N}
$$

The training observations $\boldsymbol{y}$ based on $N_{T}$ observables are in the space

$$
Y \triangleq \mathbb{R}^{N_{T}}
$$

The potential function $V$ is identified by its difference to the harmonic potential $V_{o} \triangleq \frac{1}{2} x^{2}$, thus 
we define the space containing all admissible potential functions as

$$
W^{\mathbf{1}} \triangleq\left\{V:\left(V-\frac{1}{2} x^{2}\right) \in L^{\infty}(\mathbb{R}) \cap L^{2}(\mathbb{R})\right\} .
$$

Since the Hermite basis function $\left\{\phi_{i}\right\}$ (where $\phi_{i} \in L^{2}(\mathbb{R})$, see Section A in Appendix for definition) is a complete orthogonal basis for $L^{2}(\mathbb{R})$, any given element $V \in W^{\mathbf{1}}$ can be written as the linear combination of the basis as

$$
V=V_{o}+\sum_{i=0}^{\infty} v_{i} \phi_{i}
$$

where $v_{i}=\left\langle V-V_{o}, \phi_{i}\right\rangle_{L^{2}}$ is the coefficient of the $i$-th basis. By Eqn. 11, we have a mapping from the space of weight sequences $\left\{v_{i}\right\}$ to $W^{\mathbf{1}}$.

To make $W^{\mathbf{1}}$ a Banach space, we endow it with norm

$$
\|V\|_{W^{1}} \triangleq\left\|V-V_{o}\right\|_{L^{2}}+\left\|V-V_{o}\right\|_{L^{\infty}}
$$

Then $W^{\mathbf{1}}$ is complete (see Lem. 12 for proof).

In the numerical implementation, we use the truncated version of $W^{1}$, defined as

$$
W_{L}^{1} \triangleq\left\{V:\left(V-V_{o}\right) \in \operatorname{span}\left(\phi_{0}, \ldots \phi_{L}\right)\right\}
$$

Therefore, there exists a projection $\Pi_{L}^{1}$ of truncation from $W^{\mathbf{1}}$ to $W_{L}^{1}$, namely

$$
\Pi_{L}^{1}(V)=V_{o}+\sum_{i=0}^{L}\left\langle V-V_{o}, \phi_{i}\right\rangle \phi_{i} .
$$

Now $X, Y, W^{\mathbf{1}}$ are Banach spaces endowed with proper norms. To further discuss the relation between variables on these spaces, we need to define a few measures as the prior and posterior distributions.

According to Eqn. 5) a given potential function $V: \mathbb{R} \rightarrow \mathbb{R}$ (recall the dimension $d$ of the physical space is assumed to be 1) induces a formal Gibbs distribution $\pi^{V}(d \mathfrak{q})$ on the position configuration space $X=\mathcal{L} \mathbb{R}$. Especially, we denote $\pi^{V_{o}}$ as $\pi_{0}$. The connection between $\pi_{0}$ and $\pi^{V}$ is can be derived from the action $S(\mathfrak{q})=\int_{0}^{\beta}\left[\frac{M}{2}|\dot{\mathfrak{q}}|^{2}+V(\mathfrak{q}(\tau))\right] d \tau$, i.e.

$$
\pi^{V}(d \mathfrak{q})=\frac{1}{\mathcal{Z}^{V}} \pi_{0}(d \mathfrak{q}) \exp \left(-\int_{0}^{\beta} \stackrel{\circ}{V} \circ \mathfrak{q} d \tau\right)
$$

where we use the shorthand notation $\stackrel{\circ}{V} \triangleq V-V_{o}=V-\frac{1}{2} x^{2}$ and $\mathcal{Z}^{V} \triangleq \int_{X} \pi_{0}(d \mathfrak{q}) \exp \left(-\int_{0}^{\beta} \stackrel{\circ}{V} \circ \mathfrak{q} d \tau\right)$ is the normalization constant.

We regard the measure $\pi_{0}$ induced by the harmonic oscillation potential $V_{o}$ as the prior on $X$, while $\pi^{V}$ is the posterior distribution which is absolutely continuous to $\pi_{0}$. The Radon-Nikodym (R-N for short) derivative $\frac{1}{\mathcal{Z}^{V}} \exp \left(-\int_{0}^{\beta} \stackrel{\circ}{V} \circ \mathfrak{q} d \tau\right)$ can be viewed as a 'correction' to the prior, adding more weight where the correction potential $\stackrel{\circ}{V}$ is low. 
To analyze the stability in the numerical algorithm, we can define the Gibbs distribution in the ring polymer representation in a similar way. By Eqn. 3 , a Gibbs distribution $\pi_{N}^{V}(\boldsymbol{q})$ on $X_{N}=\mathbb{R}^{N}$ is defined via the action $S_{N}(\boldsymbol{q})$ given the potential function $V$. Especially, we denote $\pi_{N}^{V_{o}}$ as $\pi_{0, N}$. The $\mathrm{R}-\mathrm{N}$ derivative of $\pi_{N}^{V}$ with respect to $\pi_{0, N}$ is

$$
\frac{\mathrm{d} \pi_{N}^{V}}{\mathrm{~d} \pi_{0, N}}(\boldsymbol{q})=\frac{1}{\mathcal{Z}_{N}^{V}} \exp \left(-\beta_{N} \sum_{i=1}^{N} \stackrel{\circ}{V}\left(q_{i}\right)\right)
$$

where $\mathcal{Z}_{N}^{V}$ is the normalization constant.

To give a prior distribution on the potential function space $W^{\mathbf{1}}$, we construct each potential function $V$ from its component $v_{i}$ in the way that

$$
v_{i}=\gamma_{i} \xi_{i}, \stackrel{i . i . d .}{\sim} \mathcal{N}(0,1)
$$

where $\left\{\gamma_{i}\right\}$ is a fixed decaying sequence. This form naturally leads to a Gaussian measure $\mu_{0}(d V)$ on $W^{\mathbf{1}}$, which is defined by the mean value $\stackrel{\circ}{V}_{o}=0$ and the covariance operator

$$
\Gamma_{V} \triangleq \sum_{n=0}^{\infty} \gamma_{i}^{2} \phi_{i} \otimes \phi_{i}
$$

The correspondence in ring polymer representation is the truncated covariance operator

$$
\Gamma_{V, L} \triangleq \sum_{n=0}^{L} \gamma_{i}^{2} \phi_{i} \otimes \phi_{i}
$$

Now we turn to the space of training observations $Y$. Assuming that the noise $\boldsymbol{\eta}$ is a Gaussian noise with mean $\mathbf{0}$ and positive-definite covariance matrix $\Gamma_{\boldsymbol{\eta}} \in \mathbb{R}^{N_{T} \times N_{T}}$, the distribution of $\boldsymbol{\eta}$ is clearly $\mathcal{N}\left(\mathbf{0}, \Gamma_{\boldsymbol{\eta}}\right)$. By the additive noise assumption, the training observation $\boldsymbol{y}$ is a translation of the ground truth $G^{A}(V)$, so the distribution of $\boldsymbol{y}$ is $\mathcal{N}\left(G^{A}(V), \Gamma_{\boldsymbol{\eta}}\right)$ if the potential function $V$ is fixed. To sum up, we denote $\tau_{0} \triangleq \mathcal{N}\left(\mathbf{0}, \Gamma_{\boldsymbol{\eta}}\right)$ as the noise distribution and $\tau^{V} \triangleq \mathcal{N}\left(G^{A}(V), \Gamma_{\boldsymbol{\eta}}\right)$ as the shifted distribution dependent on $Y$.

\subsection{Formulation: the inversion process}

Recall that the goal is to sample the potential function $V$ as well as the testing observable. As we have stated in the beginning of this section, it is equivalent to obtain the weighted average either by sampling $G^{O}(V)$ from $\mu^{\boldsymbol{y}}(d V)$ (i.e. the algorithm's perspective) or by sampling $\bar{O}[\mathfrak{q}]$ from $\pi^{\boldsymbol{y}}(d \mathfrak{q})$ (i.e. viewing $V$ as an intermediate variable). To describe the posterior distribution $\pi^{\boldsymbol{y}}(d \mathfrak{q})$, it is essential to start from the two conditional variables $V \mid \boldsymbol{y}$ and $\mathfrak{q} \mid \boldsymbol{y}$. In the following reasoning, we establish the posterior distribution $\mu^{\boldsymbol{y}}(d V)$ of $V \mid \boldsymbol{y}$ in Eqn. 17 and show that the posterior measure for $\mathfrak{q} \mid \boldsymbol{y}$ is

$$
p(\mathfrak{q} \mid \boldsymbol{y}) d \mathfrak{q}=\int_{W^{1}}\left[\mu^{\boldsymbol{y}}(d V) \pi^{V}(d \mathfrak{q})\right] .
$$


Recall that $V: \mathbb{R} \rightarrow \mathbb{R}$ is the potential function (which is in $W^{\mathbf{1}}$ ), $\boldsymbol{y} \in \mathbb{R}^{N_{T}}$ is the training observation and $\mathfrak{q} \in \mathcal{L} \mathbb{R}$ is the configuration. Thus, $\mathfrak{q} \mid V$ corresponds to the configuration variable conditioned on the given potential function of the system while $\boldsymbol{y} \mid V$ corresponds to the conditioned thermal average of training observables.

As we have assumed, the training observation $\boldsymbol{y}$ is a noisy version of the exact value $G^{A}(V)$. Recall that, the noise $\boldsymbol{\eta}$ is independent of $V$ and the distribution of $\boldsymbol{\eta}$ is $\tau_{0}=\mathcal{N}\left(\mathbf{0}, \Gamma_{\boldsymbol{\eta}}\right)$, so according to Eqn. 10. the conditional distribution of $\boldsymbol{y} \mid V$ is

$$
\tau^{V}=\mathcal{N}\left(G^{A}(V), \Gamma_{\boldsymbol{\eta}}\right)
$$

merely a translation of $\tau_{0}$.

Consider the joint probability density for $(\mathfrak{q}, \boldsymbol{y}, V)$ :

$$
\begin{aligned}
p(\mathfrak{q}, \boldsymbol{y}, V) & =p(\mathfrak{q}, \boldsymbol{y} \mid V) p(V) \\
& =p(\mathfrak{q} \mid V) p(\boldsymbol{y} \mid V) p(V)
\end{aligned}
$$

the second equation holds since the noise $\eta$ is assumed independent from the configuration $\mathfrak{q}$, thus the two conditional variable $\mathfrak{q} \mid V$ and $\boldsymbol{y} \mid V$ are independent. So by using the Bayes's formula at the following starred equation, we have

$$
\begin{aligned}
p(\mathfrak{q}, V \mid \boldsymbol{y}) & =\frac{p(\mathfrak{q}, \boldsymbol{y}, V)}{p(\boldsymbol{y})} \\
& =p(\mathfrak{q} \mid V) \frac{p(\boldsymbol{y} \mid V) p(V)}{p(\boldsymbol{y})} \\
& \stackrel{*}{=} p(\mathfrak{q} \mid V) p(V \mid \boldsymbol{y}) .
\end{aligned}
$$

By taking integrals on both sides of Eqn. 14 on $W^{1}$, we have

$$
\begin{aligned}
p(\mathfrak{q} \mid \boldsymbol{y}) & =\int_{W^{\mathbf{1}}} p(\mathfrak{q}, V \mid \boldsymbol{y}) d V \\
& =\int_{W^{\mathbf{1}}} p(\mathfrak{q} \mid V) p(V \mid \boldsymbol{y}) d V .
\end{aligned}
$$

By Eqn. 12 the potential function $V$, as a random variable in the measurable space $W^{\mathbf{1}}$, induces a probability measure $\pi^{V}$ on the configuration space $X$ for the conditional random variable $\mathfrak{q} \mid V$. For $V \mid \boldsymbol{y}$, since the R-N derivative for $\tau^{V}$ with respect to $\tau_{0}$ is

$$
\frac{d \tau^{V}}{d \tau_{0}}(\boldsymbol{y})=\frac{\exp \left(-\frac{1}{2}\left\|\Gamma_{\boldsymbol{\eta}^{-\frac{1}{2}}}\left(\boldsymbol{y}-G^{A}(V)\right)\right\|_{2}^{2}\right)}{\exp \left(-\frac{1}{2}\left\|\Gamma_{\boldsymbol{\eta}}^{-\frac{1}{2}} \boldsymbol{y}\right\|_{2}^{2}\right)}
$$

thus the negative log likelihood can be defined as

$$
\Phi(V ; \boldsymbol{y}) \triangleq \frac{1}{2}\left\|\Gamma_{\boldsymbol{\eta}^{-\frac{1}{2}}}\left(\boldsymbol{y}-G^{A}(V)\right)\right\|_{2}^{2}-\frac{1}{2}\left\|\Gamma_{\boldsymbol{\eta}}^{-\frac{1}{2}} \boldsymbol{y}\right\|_{2}^{2}=-\log \left(\frac{d \tau^{V}}{d \tau_{0}}(\boldsymbol{y})\right) .
$$


To utilize Thm. 20, we develop the following argument. Denote $\nu_{0}$ to be the product measure $\nu_{0}(\mathrm{~d} V, \mathrm{~d} \boldsymbol{y}) \triangleq \mu_{0}(\mathrm{~d} V) \tau_{0}(\mathrm{~d} \boldsymbol{y})$ and assume the negative log likelihood $\Phi$ is $\nu_{0}$-measurable. Since the conditional variable $\boldsymbol{y} \mid V$ is distributed according to $\tau^{V}(\mathrm{~d} \boldsymbol{y})$, the random variable pair $(V, y)$ is distributed according to $\nu(\mathrm{d} V, \mathrm{~d} \boldsymbol{y}) \triangleq \mu_{0}(\mathrm{~d} V) \tau^{V}(\mathrm{~d} \boldsymbol{y})$. Furthermore, we have $\nu \ll \nu_{0}$ with

$$
\frac{\mathrm{d} \nu}{\mathrm{d} \nu_{0}}(V, \boldsymbol{y})=\exp (-\Phi(V ; \boldsymbol{y})) .
$$

Since $\nu_{0}$ is a product measure, the conditional distribution for $V \mid \boldsymbol{y}$ under $\nu_{0}$ naturally exists and equals $\mu_{0}$. Thus, by Thm. 20, the conditional distribution of $V \mid \boldsymbol{y}$ under $\nu$ is

$$
\mu^{\boldsymbol{y}}(d V) \triangleq \mu_{0}(d V) \frac{1}{Z(\boldsymbol{y})} \exp (-\Phi(V ; \boldsymbol{y}))
$$

where $Z(\boldsymbol{y}) \triangleq \int_{W^{1}} \exp (-\Phi(V ; \boldsymbol{y})) \mu_{0}(d V)$ is the partition function. $\mu^{\boldsymbol{y}}$ is also called the posterior distribution for $V \mid \boldsymbol{y}$.

To sum up, we denote $\pi^{\boldsymbol{y}}$ as the posterior distribution of $\mathfrak{q} \mid \boldsymbol{y}$ obtained from 16 and 17 , i.e.

$$
\pi^{\boldsymbol{y}}(d \mathfrak{q})=\int_{W^{1}}\left[\mu^{\boldsymbol{y}}(d V) \pi^{V}(d \mathfrak{q})\right] .
$$

As we have assumed, the posterior distributions $\mu^{\boldsymbol{y}}$ and $\pi^{V}$ are absolutely continuous with respect to the prior distributions $\mu_{0}$ and $\pi_{0}$ correspondingly, so the absolute continuity of $\pi^{y}$ with respect to $\pi_{0}$ holds from Eqn. 18, Moreover, we give the R-N derivative as follows without proof.

Lemma 1. The $R-N$ derivative of the posterior distribution $\pi^{\boldsymbol{y}}$ w.r.t. the prior distribution $\pi_{0}$ is

$$
\frac{d \pi^{\boldsymbol{y}}}{d \pi_{0}}(\mathfrak{q})=\int_{W^{1}}\left[\frac{d \mu^{\boldsymbol{y}}}{d \mu_{0}}(V) \frac{d \pi^{V}}{d \pi_{0}}(\mathfrak{q}) \mu_{0}(d V)\right] .
$$

With these posterior distribution established, now we can examine the coherence between our model (sampling $\bar{O}[\mathfrak{q}]$ from $\pi^{\boldsymbol{y}}(d \mathfrak{q})$ ) and algorithm (sampling $G^{O}(V)$ from $\mu^{\boldsymbol{y}}(d V)$ ) by the following proposition.

Proposition 2. The following two weighted averages are the same:

$$
\mathbb{E}_{\mu^{y}(d V)} G^{O}(V)=\mathbb{E}_{\pi^{y}(d \mathfrak{q})} \bar{O}[\mathfrak{q}]
$$

Proof. The proof requires no more than a direct calculation: by definition of $\mu^{\boldsymbol{y}}(d V)$ and $G^{O}(V)$ we have

$$
\mathbb{E}_{\mu^{y}(d V)} G^{O}(V)=\int_{W^{1}} \mu^{\boldsymbol{y}}(d V)\left[\int_{X} \pi^{V}(d \mathfrak{q}) \bar{O}[\mathfrak{q}]\right] .
$$


On the other hand, by interchanging the order of integrals and definition of $\pi^{\boldsymbol{y}}(d \mathfrak{q})$, we have

$$
\begin{aligned}
\int_{W^{1}} \mu^{\boldsymbol{y}}(d V)\left[\int_{X} \pi^{V}(d \mathfrak{q}) \bar{O}[\mathfrak{q}]\right] & =\int_{X} \bar{O}[\mathfrak{q}]\left[\int_{W^{1}} \mu^{\boldsymbol{y}}(d V) \pi^{V}(d \mathfrak{q})\right] \\
& =\int_{X} \bar{O}[\mathfrak{q}] \pi^{\boldsymbol{y}}(d \mathfrak{q}) \\
& =\mathbb{E}_{\pi^{y}(d \mathfrak{q})} \bar{O}[\mathfrak{q}] .
\end{aligned}
$$

So we arrive at $\mathbb{E}_{\mu^{y}(d V)} G^{O}(V)=\mathbb{E}_{\pi^{y}(d \mathfrak{q})} \bar{O}[\mathfrak{q}]$.

At the end of this section, we write down the corresponding conclusion when $\boldsymbol{q} \in X_{N}=\mathbb{R}^{N}$ is a discrete ring under the ring polymer representation and $V \in W_{L}^{\mathbf{1}}$ is in the truncated potential space. Denote $\mu_{0, L}$ as the marginal measure of $\mu_{0}$ on the subspace $W_{L}^{1}$. The conditional distribution of $V \mid \boldsymbol{y}$ on the subspace $W_{L}^{\mathbf{1}}$ can be similarly defined as

$$
\mu_{L, N}^{\boldsymbol{y}}(d V) \triangleq \mu_{0, L}(d V) \frac{1}{Z_{L, N}(\boldsymbol{y})} \exp \left(-\Phi_{N}(V ; \boldsymbol{y})\right)
$$

where $Z_{L, N}(\boldsymbol{y}) \triangleq \int_{W_{L}^{1}} \mu_{0, L}(d V) \exp \left(-\Phi_{N}(V ; \boldsymbol{y})\right)$ is the partition function and

$$
\Phi_{N}(V ; \boldsymbol{y}) \triangleq \frac{1}{2}\left\|\Gamma_{\boldsymbol{\eta}}^{-\frac{1}{2}}\left(\boldsymbol{y}-G_{N}^{A}(V)\right)\right\|_{2}^{2}-\frac{1}{2}\left\|\Gamma_{\boldsymbol{\eta}}^{-\frac{1}{2}} \boldsymbol{y}\right\|_{2}^{2}
$$

is the negative log likelihood with consideration of the ring polymer approximation. Since the posterior distribution of $\boldsymbol{q} \mid \boldsymbol{y}$ depends both on the bead number $N$ and the truncation level $L$, we denote it as $\pi_{L, N}^{y}$. A similar computation gives

$$
\pi_{L, N}^{\boldsymbol{y}}(d \mathfrak{q})=\int_{W_{L}^{1}}\left[\mu_{L, N}^{\boldsymbol{y}}(d V) \pi_{N}^{V}(d \mathfrak{q})\right]
$$

Two similar conclusions are listed below without proof.

Lemma 3. The $R$ - $N$ derivative of the posterior distribution $\pi_{N}^{\boldsymbol{y}}$ w.r.t. the prior distribution $\pi_{0, N}$ is

$$
\frac{d \pi_{L, N}^{\boldsymbol{y}}}{d \pi_{0, N}}(\boldsymbol{q})=\int_{W_{L}^{1}}\left[\frac{d \mu_{L, N}^{\boldsymbol{y}}}{d \mu_{0, L}}(V) \frac{d \pi_{N}^{V}}{d \pi_{0, N}}(\mathfrak{q}) \mu_{0, L}(d V)\right] .
$$

Proposition 4. The following two weighted averages are the same:

$$
\mathbb{E}_{\mu_{L, N}^{y}(d V)} G_{N}^{O}(V)=\mathbb{E}_{\pi_{L, N}^{y}(d \boldsymbol{q})} \bar{O}[\boldsymbol{q}] .
$$




\section{The Inversion Algorithm}

In this section, we will introduce an inversion algorithm for the thermal average sampling in the single level quantum system. Recall that our interest lies in the conditional variable $\mathfrak{q} \mid \boldsymbol{y}$, described by

$$
p(\mathfrak{q} \mid \boldsymbol{y})=\int_{W^{1}} p(\mathfrak{q} \mid V) p(V \mid \boldsymbol{y}) d V
$$

in which the potential $V$ is viewed as an intermediate variable. Therefore, by making use of the PIMD method (Sec. 2) to sample $\mathfrak{q} \mid V$ and the posterior distribution sampler (Sec. C.4 in Appendix) to sample $V \mid \boldsymbol{y}$, the inversion algorithm can sample the conditional variable $\mathfrak{q} \mid \boldsymbol{y}$ as well as the testing observation $G^{O}(V) \mid \boldsymbol{y}$.

\subsection{Algorithm overview}

The algorithm is mainly two-staged: the first stage is to sample a fixed potential function $\widehat{V}^{(k)}$ proposed by the prior distribution and obtain the $k$-th training thermal average $\boldsymbol{y}^{(k)}=G^{A}\left(\widehat{V}^{(k)}\right)$ based on path integral molecular dynamics; at the second stage, the average $\boldsymbol{y}^{(k)}$ is compared against the ground truth $\boldsymbol{y}^{*}$ and previous sample $\boldsymbol{y}^{(k-1)}$, where Metropolis-Hasting method is used to decide whether to keep the proposed $\widehat{V}^{(k)}$ or not. By this way, the conditional variable $V \mid \boldsymbol{y}^{*}$ can be sampled accurately up to the error in PIMD simulations.

\subsection{Algorithm implementation}

\subsubsection{PIMD solver (Line 5 in Alg. 1)}

To efficiently solve the forward problem, we implement a PIMD solver in $\mathrm{C}++$ and perform tests on a machine equipped with a Intel Core i5-7300HQ (single-threaded at $3.5 \mathrm{GHz}$ ).

\subsubsection{Metropolis Hasting method (Line 10 in Alg. 1)}

The measure of interest $\mu^{y}$ is absolutely continuous with respect to $\mu_{0}$ and its R-N derivative is in proportion to $\exp (-\Phi(V ; \boldsymbol{y}))$. So in order to sample $\mu^{\boldsymbol{y}}$ based on proposals from $\mu_{0}$, according to Assu. 24, the decision function can be chosen as

$$
a\left(V_{\text {old }} \rightarrow V_{\text {new }}\right)=\exp \left(\min \left\{0, \Phi\left(V_{\text {old }}\right)-\Phi\left(V_{\text {new }}\right)\right\}\right)
$$

so that the detailed balance condition will be satisfied as long as the proposal kernel is reversible with respect to the prior distribution. 


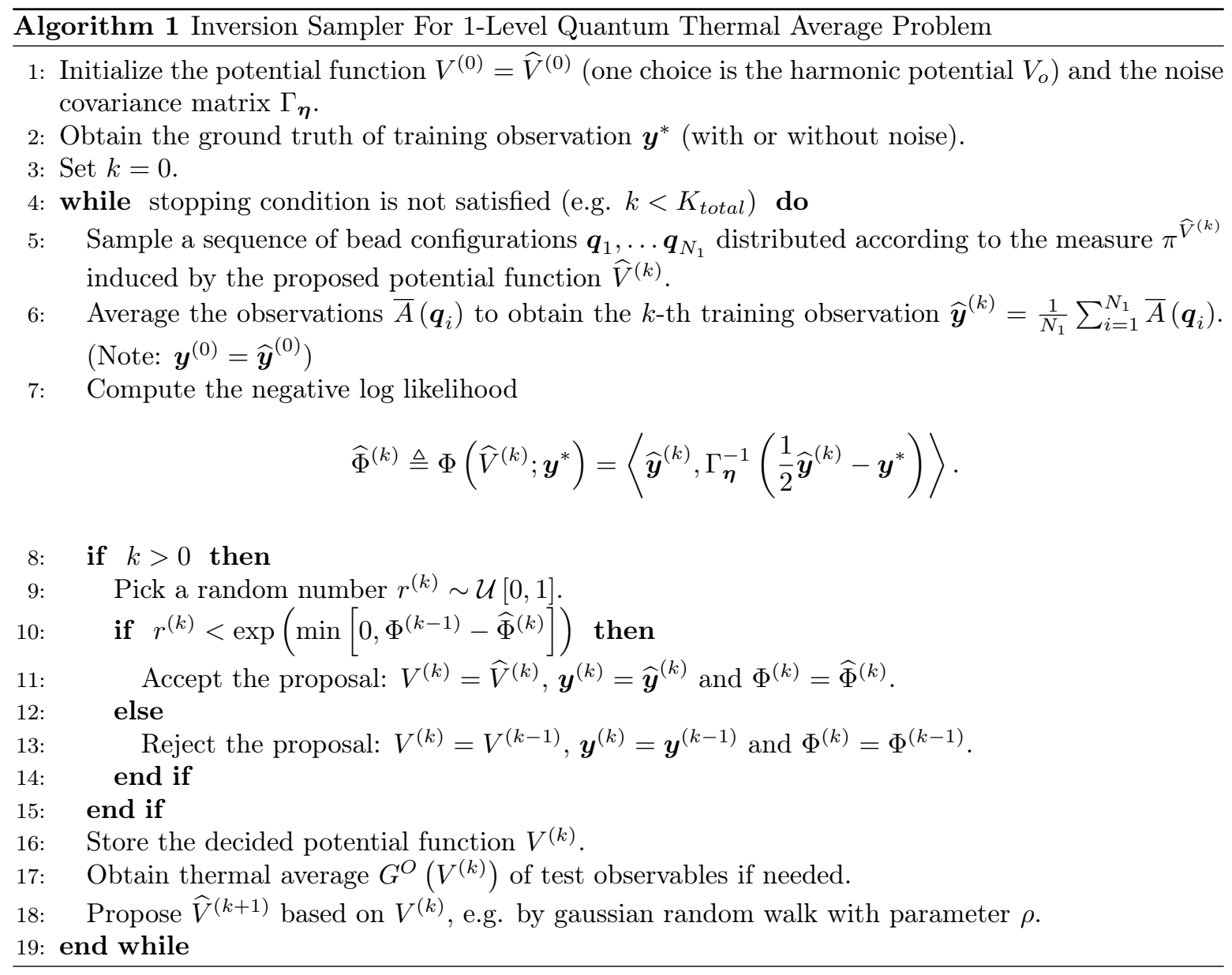




\subsubsection{Proposal kernel of potential function (Line 18 in Alg. 1)}

The next step is to choose a proposal kernel which is reversible with respect to the prior distribution. Recall that each potential function $V$ in the truncated space $W_{L}^{1}$ can be expressed as

$$
V=V_{o}+\sum_{i=0}^{L} \xi_{i} \gamma_{i} \phi_{i}
$$

while the covariance operator (defined in Eqn. 3.1) has the matrix form $\operatorname{diag}\left(\gamma_{0}^{2}, \ldots \gamma_{L}^{2}\right)$, so to propose $V$ is essentially to propose $\left\{\xi_{i}\right\}_{i=0}^{L}$.

To avoid high rejection rate, we adopt the gaussian random walk (in Cotter et al., 2012 also called "pre-conditioned CN proposal") in the proposal stage: given a gaussian random variable $r$ and parameter $0<\rho<1$, then by generating an independent standard gaussian variable $g \sim \mathcal{N}(0,1)$ we obtain

$$
r^{*}=\rho r+\sqrt{1-\rho^{2}} g
$$

which shares the same mean and variance with $r$. Moreover, the correlation between $r^{*}$ and $r$ is exactly $\rho$, so a larger $\rho$ can make two successive proposals closer while keeping the desired invariant distribution unchanged.

In the 2-level problem we also need to propose r.v. that follow the exponential distribution. Based on the observation that the sum of squares of two standard gaussian variables follows the exponential distribution, we can prepare two standard gaussian variable $g_{1}, g_{2} \sim \mathcal{N}(0,1)$ and use the given exponential variable $r$ and parameter $0<\rho<1$ to obtain

$$
r^{*}=\left(\rho \sqrt{r}+\bar{\rho} g_{1}\right)^{2}+\left(\bar{\rho} g_{2}\right)^{2}, \bar{\rho} \triangleq \sqrt{\frac{1-\rho^{2}}{2}},
$$

which also shares the same mean and variance with $r$ and has a $\rho$ correlation with $r$.

\subsubsection{Regularity of $V \in W^{1}$}

Recall that elements in $W^{\mathbf{1}}$ can be expressed in the form of

$$
V=V_{o}+\sum_{i=0}^{\infty} \xi_{i} \gamma_{i} \phi_{i}
$$

where $\left\{\xi_{i}\right\}$ are the random variables and $\left\{\phi_{i}\right\}$ is the basis. In particular, the decaying sequence $\left\{\gamma_{i}\right\}$ serves as a parameter controlling the regularity of $V$. We assume that, given a constant parameter $\beta \in(0,1)$, the asymptotic behavior of $\left\{\gamma_{i}\right\}$ is given by

$$
\gamma_{i}=\mathcal{O}\left(i^{-s}\right), s>\max \left\{1, \frac{\beta+2}{4(1-\beta)}\right\}
$$

Under such assumption, we have the following properties:

1. The exponent $s$ is larger than 1, so by Prop. 15, $V-V_{o}$ is in $L^{\infty} \cap L^{2}$ and $V$ is in $W^{1}$. 
2. The exponent $s$ is larger than $\frac{\beta+2}{4(1-\beta)}$, so by Prop. $16, V$ has $\beta$-order Hölder continuity.

\section{$5 \quad$ Stability Analysis}

In terms of stability, recall that the variable of interest is $\mathfrak{q} \mid \boldsymbol{y}$ in distribution of $\pi^{\boldsymbol{y}}$, so we want to establish a continuous dependency of $\pi^{\boldsymbol{y}}$ on $\boldsymbol{y}$. Targeted at this, the proof is structured in the following manner. First, Lem. 5 shows that the induced measure $\pi^{V}$ on configuration space continuously depends on the potential $V$, based on which Cor. 6 ensures that the thermal average $G^{A}(V)$ is a continuous with respect to $V$. Then Lem. 7 shows that the induced measure $\mu^{y}$ on potential space continuously depends on the training observation $\boldsymbol{y}$. The main theorem (Thm. 8) utilizes the two lemmas and the formula for $\pi^{y}$ to control the perturbation between $\pi^{y}$ by that between $\mu^{\boldsymbol{y}}$, leading to the conclusion. Finally, Cor. 9 shows how to bound the perturbation in testing observations by that in training observations.

Notation. Given a separable Banach space $\mathcal{X}$ and two functions $f_{1}, f_{2}$ on $\mathcal{X} \times \mathcal{X}$. We call $f_{1} \lesssim_{r} f_{2}$ if, for every fixed $r>0$, there is $C=C(r)>0$ such that

$$
f_{1}\left(x, x^{\prime}\right) \leq C f_{2}\left(x, x^{\prime}\right), \forall x, x^{\prime} \in B_{\mathcal{X}}(0, r)
$$

Lemma 5. Given two potential functions $V_{1}, V_{2} \in W^{\mathbf{1}}$, we have

$$
d_{H e l l}\left(\pi^{V_{1}}, \pi^{V_{2}}\right) \lesssim_{r}\left\|V_{1}-V_{2}\right\|_{W^{1}}
$$

where the definition of Hellinger distance is given by Def. 18 in the Appendix.

The proof of this lemma is a direct application of Thm. 22 in the Appendix.

Proof. First we check the conditions for Assu. 21. notice that $\Phi(\mathfrak{q} ; V)=\int_{0}^{\beta} \stackrel{\circ}{V} \circ \mathfrak{q} d \tau$ is continuous as a function of $\mathfrak{q}$, and that

$$
\begin{gathered}
\Phi(\mathfrak{q} ; V) \geq-\beta\|\stackrel{V}{V}\|_{L^{\infty}} \geq-\beta\|V\|_{W^{1}}, \\
\left|\Phi\left(\mathfrak{q} ; V_{1}\right)-\Phi\left(\mathfrak{q} ; V_{2}\right)\right|=\left|\int_{0}^{\beta}\left(V_{1}-V_{2}\right) \circ \mathfrak{q} d \tau\right| \leq \beta\left\|V_{1}-V_{2}\right\|_{\infty} \leq \beta\left\|V_{1}-V_{2}\right\|_{W^{1}},
\end{gathered}
$$

thus we can take $M_{1}(r)=\beta r$ and $M_{2}=\beta$.

Since $M_{1}$ and $M_{2}$ are constant with respect to $\mathfrak{q}$, by Thm. 22, there exists $C=C(r)$ such that for all $V_{1}, V_{2} \in B_{W^{1}}(0, r)$,

$$
d_{H e l l}\left(\pi^{V_{1}}, \pi^{V_{2}}\right) \leq C\left\|V_{1}-V_{2}\right\|_{W^{1}} .
$$

Corollary 6. The ensemble average of a given bounded observable $A$ is continuous as a function of the potential function, i.e.

$$
\left|G^{A}\left(V_{1}\right)-G^{A}\left(V_{2}\right)\right| \lesssim_{r}|| V_{1}-V_{2} \|_{W^{1}}
$$

where $G^{A}(V) \triangleq \mathbb{E}_{\pi^{V}(d \mathfrak{q})} \frac{1}{\beta} \int_{0}^{\beta} A \circ \mathfrak{q} d \tau=\mathbb{E}_{\pi^{V}(d \mathfrak{q})} \bar{A}[\mathfrak{q}]$. 
Proof. This is a direct corollary of Lem. 23 and Lem. 5 since $G^{A}(V)$ is a bounded function on $W^{\mathbf{1}}$.

Lemma 7. Given two training observations $\boldsymbol{y}_{1}, \boldsymbol{y}_{2}$ and assume the training observable $A$ is bounded, we have

$$
d_{H e l l}\left(\mu^{\boldsymbol{y}_{1}}, \mu^{\boldsymbol{y}_{2}}\right) \lesssim_{r}\left\|\boldsymbol{y}_{1}-\boldsymbol{y}_{2}\right\|_{2}
$$

where $\|\cdot\|_{2}$ is the Euclidean norm on $\mathbb{R}^{N_{T}}$ and the Hellinger distance is given by Def. 18 .

The proof of this lemma is another direct application of Thm. 22

Proof. Again we check the conditions for Assu. 21. notice that

$$
\Phi(V ; \boldsymbol{y})=\frac{1}{2}\left\|\Gamma_{\boldsymbol{\eta}}^{-\frac{1}{2}}\left(\boldsymbol{y}-G^{A}(V)\right)\right\|_{2}^{2}-\frac{1}{2}\left\|\Gamma_{\boldsymbol{\eta}^{-\frac{1}{2}}} \boldsymbol{y}\right\|_{2}^{2}
$$

is continuous as a function of the two variables $(V, \boldsymbol{y})$ (by Cor. 6), and that

$$
\begin{gathered}
\Phi(V ; \boldsymbol{y}) \geq-\frac{1}{2}\left\|\Gamma_{\eta}^{-\frac{1}{2}} \boldsymbol{y}\right\|_{2}^{2} \\
\left|\Phi\left(V ; \boldsymbol{y}_{1}\right)-\Phi\left(V ; \boldsymbol{y}_{2}\right)\right| \leq\left[\frac{1}{2}\left\|\boldsymbol{y}_{1}+\boldsymbol{y}_{2}\right\|_{2}+\|A\|_{\infty}\right]\left\|\Gamma_{\boldsymbol{\eta}}^{-1}\right\|_{2}\left\|\boldsymbol{y}_{1}-\boldsymbol{y}_{2}\right\|_{2},
\end{gathered}
$$

we can take $M_{1}=\frac{1}{2}\left\|\Gamma_{\boldsymbol{\eta}}^{-\frac{1}{2}} \boldsymbol{y}\right\|_{2}^{2}$ and $M_{2}(r)=\left\|\Gamma_{\boldsymbol{\eta}}^{-1}\right\|_{2}\left(r+\|A\|_{\infty}\right)$, where $\left\|\Gamma_{\boldsymbol{\eta}}^{-1}\right\|_{2}$ is the operator norm induced by the vector norm $\|\cdot\|_{2}$. Since they are also constant with respect to $V$, by Thm. 22 , there is $C=C(r)$ such that for all $\boldsymbol{y}_{1}, \boldsymbol{y}_{2} \in B_{Y}(0, r)$,

$$
d_{H e l l}\left(\mu^{\boldsymbol{y}_{1}}, \mu^{\boldsymbol{y}_{2}}\right) \leq C\left\|\boldsymbol{y}_{1}-\boldsymbol{y}_{2}\right\|_{2} .
$$

Theorem 8. Given two training observations $\boldsymbol{y}_{1}, \boldsymbol{y}_{2}$ and assume the training observable $A$ is bounded, the distance between two induced measure on $\mathcal{L} \mathbb{R}$ can be bounded by the their distance, i.e.

$$
d_{T V}\left(\pi^{\boldsymbol{y}_{1}}, \pi^{\boldsymbol{y}_{2}}\right) \lesssim r\left\|\boldsymbol{y}_{1}-\boldsymbol{y}_{2}\right\|_{2}
$$

Proof. The proof consists of two steps: first we will bound the distance between $\pi^{\boldsymbol{y}}$ by the distance between $\mu^{\boldsymbol{y}}$, then we will compare the latter part with the distance between $\boldsymbol{y}$.

By definition of TV distance and the fact that $\pi^{y} \ll \pi_{0}$, we can expand the left-hand side as

$$
\begin{aligned}
d_{T V}\left(\pi^{\boldsymbol{y}_{1}}, \pi^{\boldsymbol{y}_{2}}\right) & =\int_{\mathcal{L} \mathbb{R}} \pi_{0}(d \mathfrak{q})\left|\frac{d \pi^{\boldsymbol{y}_{1}}}{d \pi_{0}}(\mathfrak{q})-\frac{d \pi^{\boldsymbol{y}_{2}}}{d \pi_{0}}(\mathfrak{q})\right| \\
& =\int_{\mathcal{L} \mathbb{R}} \pi_{0}(d \mathfrak{q})\left|\int_{W^{1}} \mu_{0}(d V) \frac{d \pi^{V}}{d \pi_{0}}(\mathfrak{q})\left[\frac{d \mu^{\boldsymbol{y}_{1}}}{d \mu_{0}}(V)-\frac{d \mu^{\boldsymbol{y}_{2}}}{d \mu_{0}}(V)\right]\right|,
\end{aligned}
$$

where the second equation results from the definition of $\pi^{y}$ (Eqn. 19p. By taking the absolute sign 
into the second integral and a change to the order of integral (by Tonelli's theorem), we have

$$
\begin{aligned}
d_{T V}\left(\pi^{\boldsymbol{y}_{1}}, \pi^{\boldsymbol{y}_{2}}\right) & \leq \int_{\mathcal{L} \mathbb{R}} \pi_{0}(d \mathfrak{q})\left[\int_{W^{1}} \mu_{0}(d V) \frac{d \pi^{V}}{d \pi_{0}}(\mathfrak{q})\left|\frac{d \mu^{\boldsymbol{y}_{1}}}{d \mu_{0}}(V)-\frac{d \mu^{\boldsymbol{y}_{2}}}{d \mu_{0}}(V)\right|\right] \\
& =\int_{W^{\mathbf{1}}} \mu_{0}(d V)\left[\int_{\mathcal{L} \mathbb{R}} \pi_{0}(d \mathfrak{q}) \frac{d \pi^{V}}{d \pi_{0}}(\mathfrak{q})\left|\frac{d \mu^{\boldsymbol{y}_{1}}}{d \mu_{0}}(V)-\frac{d \mu^{\boldsymbol{y}_{2}}}{d \mu_{0}}(V)\right|\right] .
\end{aligned}
$$

Notice that the R-N derivative $\frac{d \mu^{\boldsymbol{y}}}{d \mu_{0}}(V)$ does not depend on $\mathfrak{q}$, we can take the term outside the second integral:

$$
d_{T V}\left(\pi^{\boldsymbol{y}_{1}}, \pi^{\boldsymbol{y}_{2}}\right) \leq \int_{W^{\mathbf{1}}}\left\{\mu_{0}(d V)\left|\frac{d \mu^{\boldsymbol{y}_{1}}}{d \mu_{0}}(V)-\frac{d \mu^{\boldsymbol{y}_{2}}}{d \mu_{0}}(V)\right|\left[\int_{\mathcal{L} \mathbb{R}} \pi_{0}(d \mathfrak{q}) \frac{d \pi^{V}}{d \pi_{0}}(\mathfrak{q})\right]\right\} .
$$

Now we can directly calculate the second integral since $\int_{\mathcal{L} \mathbb{R}} \pi_{0}(d \mathfrak{q}) \frac{d \pi^{V}}{d \pi_{0}}(\mathfrak{q})=\int_{\mathcal{L} \mathbb{R}} \pi^{V}(d \mathfrak{q})=1$, so again by the definition of TV distance

$$
\begin{aligned}
d_{T V}\left(\pi^{\boldsymbol{y}_{1}}, \pi^{\boldsymbol{y}_{2}}\right) & \leq \int_{W^{1}} \mu_{0}(d V)\left|\frac{d \mu^{\boldsymbol{y}_{1}}}{d \mu_{0}}(V)-\frac{d \mu^{\boldsymbol{y}_{2}}}{d \mu_{0}}(V)\right| \\
& =d_{T V}\left(\mu^{\boldsymbol{y}_{1}}, \mu^{\boldsymbol{y}_{2}}\right) .
\end{aligned}
$$

By Lem. 19, we can bound the TV distance by the Hellinger distance, leading to

$$
\begin{aligned}
d_{T V}\left(\pi^{\boldsymbol{y}_{1}}, \pi^{\boldsymbol{y}_{2}}\right) \leq d_{T V}\left(\mu^{\boldsymbol{y}_{1}}, \mu^{\boldsymbol{y}_{2}}\right) & \leq \sqrt{2} d_{H e l l}\left(\mu^{\boldsymbol{y}_{1}}, \mu^{\boldsymbol{y}_{2}}\right) \\
& \lesssim r\left\|\boldsymbol{y}_{1}-\boldsymbol{y}_{2}\right\|_{2},
\end{aligned}
$$

where the second inequality holds as a result of Lem. 7

Corollary 9. Given two training observations $\boldsymbol{y}_{1}, \boldsymbol{y}_{2}$ and assume the training and testing observables $A, O$ are bounded, the difference between the expectations of the testing observable w.r.t the two posterior distributions can be bounded by the distance of training observations:

$$
\left|\mathbb{E}_{\pi \boldsymbol{y}_{1}} \bar{O}[\mathfrak{q}]-\mathbb{E}_{\pi} \boldsymbol{y}_{2} \bar{O}[\mathfrak{q}]\right| \lesssim_{r} \| \boldsymbol{y}_{1}-\left.\boldsymbol{y}_{2}\right|_{2}
$$

Proof. This is a direct corollary of Lem. 23 and Thm. 8 since $\bar{O}[\mathfrak{q}]=\frac{1}{\beta} \int_{0}^{\beta} O(\mathfrak{q}(\tau)) d \tau$ is a bounded function on $X$.

When we apply those estimates on the proposed algorithm, the bead number $N$ and the truncation level $L$ are finite, so we have to give the corresponding conclusions. However, most of the proof are the same since the only difference is the underlying measure space. Thus we list the conclusions as follows without proof:

Theorem 10. Assume the bead number $N$ and the truncation level $L$ are given and finite. Given two training observations $\boldsymbol{y}_{1}, \boldsymbol{y}_{2}$ and assume the training observable $A$ is bounded, the distance between two 
induced measure on $\mathbb{R}^{N}$ can be bounded by the their distance, i.e.

$$
d_{T V}\left(\pi_{L, N}^{\boldsymbol{y}_{1}}, \pi_{L, N}^{\boldsymbol{y}_{2}}\right) \lesssim_{r}\left\|\boldsymbol{y}_{1}-\boldsymbol{y}_{2}\right\|_{2}
$$

Remark. Normally, one expects convergence $\pi_{L, N}^{\boldsymbol{y}} \rightarrow \pi_{L, \infty}^{\boldsymbol{y}}$ with respect to $N$ (or even $\rightarrow \pi^{\boldsymbol{y}}$ when both $L$ and $N$ go to infinity), but it is not entirely clear in this case, where the approximation property relies on the rigorous justification of the path integral formulation. Whereas, if there exists a proper norm $\|\cdot\|_{*}$ on the configuration space $X=\mathcal{L} \mathbb{R}$ s.t. the following asymptotic estimation holds for every potential function $V \in W^{\mathbf{1}}$

$$
\left|S[\mathfrak{q}]-S_{N}\left(R_{N}(\mathfrak{q})\right)\right|=\left|\int_{0}^{\beta} \stackrel{\circ}{V} \circ \mathfrak{q} \mathrm{d} \tau-\beta_{N} \sum_{i=1}^{N} V\left(\mathfrak{q}\left(i \beta_{N}\right)\right)\right| \leq M\left(\|\mathfrak{q}\|_{*}\right) \psi(N)
$$

where the $R_{N}: \mathcal{L} \mathbb{R} \rightarrow \mathbb{R}^{N},\left(R_{N}(\mathfrak{q})\right)_{i}=\mathfrak{q}\left(i \beta_{N}\right)$ is the restriction operator, $M$ is a non-decreasing function and $\lim _{N \rightarrow \infty} \psi(N)=0$, then by Thm 4.9 in [Dashti and Stuart, 2017, the following convergence holds

$$
d_{\text {Hell }}\left(\pi_{N}^{V}, \pi^{V}\right) \lesssim_{r} \psi(N)
$$

Based on this we can expect $\pi_{L, N}^{\boldsymbol{y}} \rightarrow \pi_{L, \infty}^{\boldsymbol{y}}$ by estimating the error of R-N derivatives $\frac{\mathrm{d} \mu_{L, N}^{\boldsymbol{y}}}{\mathrm{d} \mu_{0, L}}$ and $\frac{\mathrm{d} \pi_{N}^{V}}{\mathrm{~d} \pi_{0, N}}$ when $N \rightarrow \infty$.

Corollary 11. Assume the bead number $N$ and the truncation level $L$ are given and finite. Given two training observations $\boldsymbol{y}_{1}, \boldsymbol{y}_{2}$ and assume the training and testing observables $A, O$ are bounded, the difference between the expectations of the testing observable w.r.t the two posterior distributions can be bounded by the distance of training observations:

$$
\mid \mathbb{E}_{\pi_{L, N}^{y_{1}} \bar{O}(\boldsymbol{q})-\mathbb{E}_{\pi_{L, N}^{\boldsymbol{y}_{2}}} \bar{O}(\boldsymbol{q}) \mid \lesssim r}\left\|\boldsymbol{y}_{1}-\boldsymbol{y}_{2}\right\|_{2}
$$

Remark. Similarly we can expect the convergence $\mathbb{E}_{\pi_{L, N}^{y_{1}}} \bar{O}\left(R_{N}(\mathfrak{q})\right) \rightarrow \mathbb{E}_{\pi_{L, \infty}^{y}} \bar{O}[\mathfrak{q}]$ with respect to $N$ (or even $\left.\rightarrow \mathbb{E}_{\pi y} \bar{O}[\mathfrak{q}]\right)$ under certain assumptions made above, which means the test prediction under ring polymer representation is consistent with that under continuum limit. We shall revisit this corollary on stability in the numerical study section.

\section{$6 \quad$ Numerical tests (1 level system)}

\subsection{Proof of concept}

First we will demonstrate that the algorithm works in a one-dimensional system setting if the training observation $\boldsymbol{y}^{*}$ is assumed without noise. The system is set-up as follows:

- Ground-truth potential function $V_{\text {truth }}=\frac{1}{2} x^{2}+5 \sin \left(\frac{5 x}{\pi}\right) \exp \left(-\frac{x^{2}}{2}\right)$, shown in Fig. 1 .

- Training observable $A_{i}=e^{-\left(x-x_{i}\right)^{2}}$ where $x_{i}=\frac{i}{2}-2, i=0 \ldots 8$, i.e. a series of Gaussians along the most probable locations. 
- Testing observable $O_{1}=\phi_{1}(2 x), O_{2}=e^{-(x+1.25)^{2}}, O_{3}=e^{-(x-0.25)^{2}}, O_{4}=\phi_{2}(3 x), O_{5}=\phi_{3}(3 x)$.

- The noise covariance matrix $\Gamma_{\eta}=\widetilde{\Gamma_{\eta}} I^{N_{1} \times N_{1}}$ with $\widetilde{\Gamma_{\eta}}=10^{-3}$

- The potential covariance sequence $\gamma_{j}=4 \cdot j^{-1.2}(j=0 \ldots 12)$.

- Particle mass $M=10$, truncation level $L=12$ and bead number $N=16$.
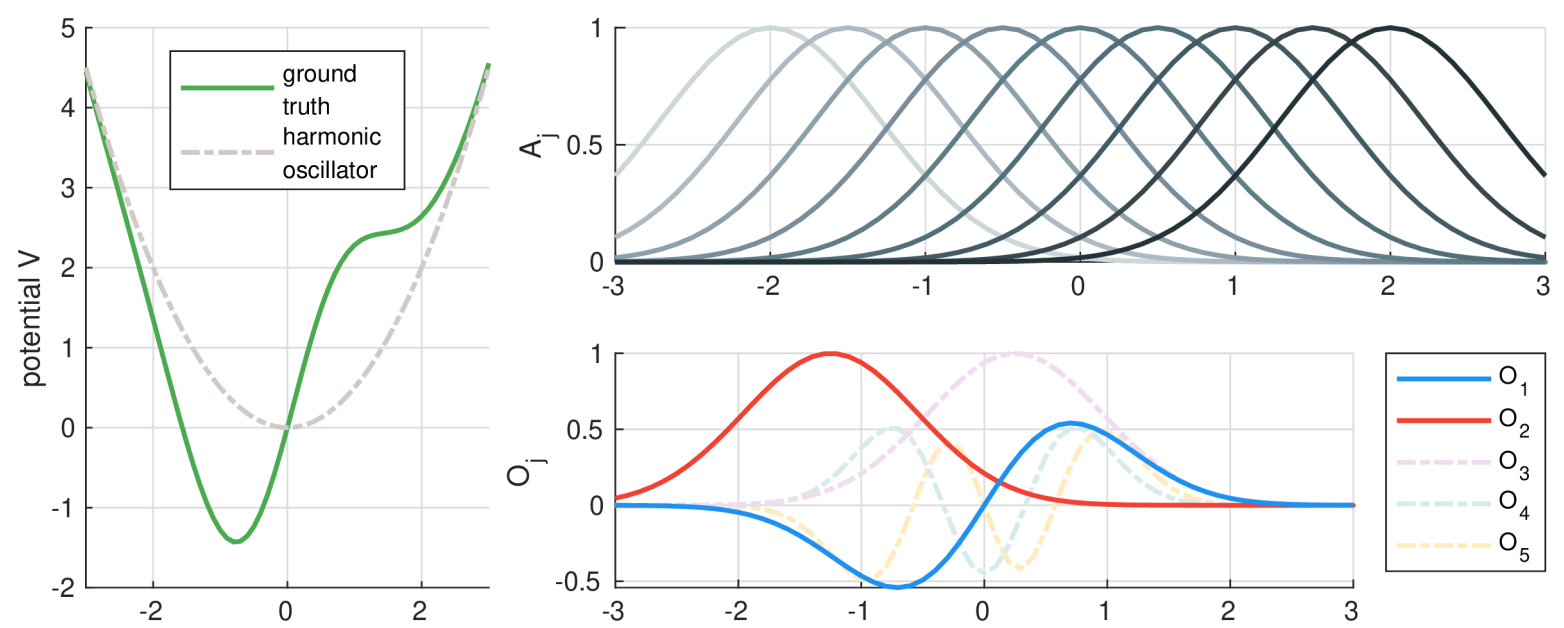

Figure 1: Left figure: the ground truth potential $V_{\text {truth }}$ (green line) is lower near $x=-1$ and higher near $x=1$ as compared to the harmonic oscillation potential (brown faded line, also used as the initial potential $\left.V^{(0)}\right)$.

Top-right figure: illustration for training observables $\left\{A_{j}\right\}_{j=0}^{8}$, each of which is a Gaussian function distributed along the most probable locations.

Bottom-right figure: illustration for testing observables $\left\{O_{j}\right\}_{j=1}^{5}$, varying in the size of support set and oscillation intensity. Among the five testing observables, the first two will be selected for demonstration since the rest three give similar results.

We ran 10 independent runs in total with 1600 proposals in each run. We select two of the five testing observables to report since the results of the rest three are similar. In the following figures, we will always label the initial guess (obtained at the first iteration, i.e. by setting the harmonic oscillation potential $V_{o}=\frac{1}{2} x^{2}$ ) as the brown dotted line and the ground truth (obtained by setting ground truth $\left.V_{\text {truth }}\right)$ as the green dashed line; we also indicate twice the standard error by the shaded area.

To ensure the algorithm indeed samples the entire landscape of the posterior distribution, we provide some plots on the statistic characteristics, where Fig. 2 shows the acceptance rate and the decrease in mean squared error and Fig. 3 shows the auto-correlation function for test observables and the coordinates $\xi_{i}$ of the sampled potential $V^{(k)}$. Since we use random gaussian walk in the proposal of $V^{(k)}$ and by default $\rho=0.95$, so we expect a large auto-correlation time. We fix $t_{a c}=50$ in the following calculations, which means we have $1600 / 50 \times 10=320$ independent samples in total.

The results of averaged sampled test observables, potential and density function are shown in Fig. 4 and 5 . Judging from the figures, the potential and density landscapes can correctly sampled where the probability density is relatively large. As for the thermal average of the testing observables, the averaged samples will converge towards the ground truth and the standard error will decrease over 

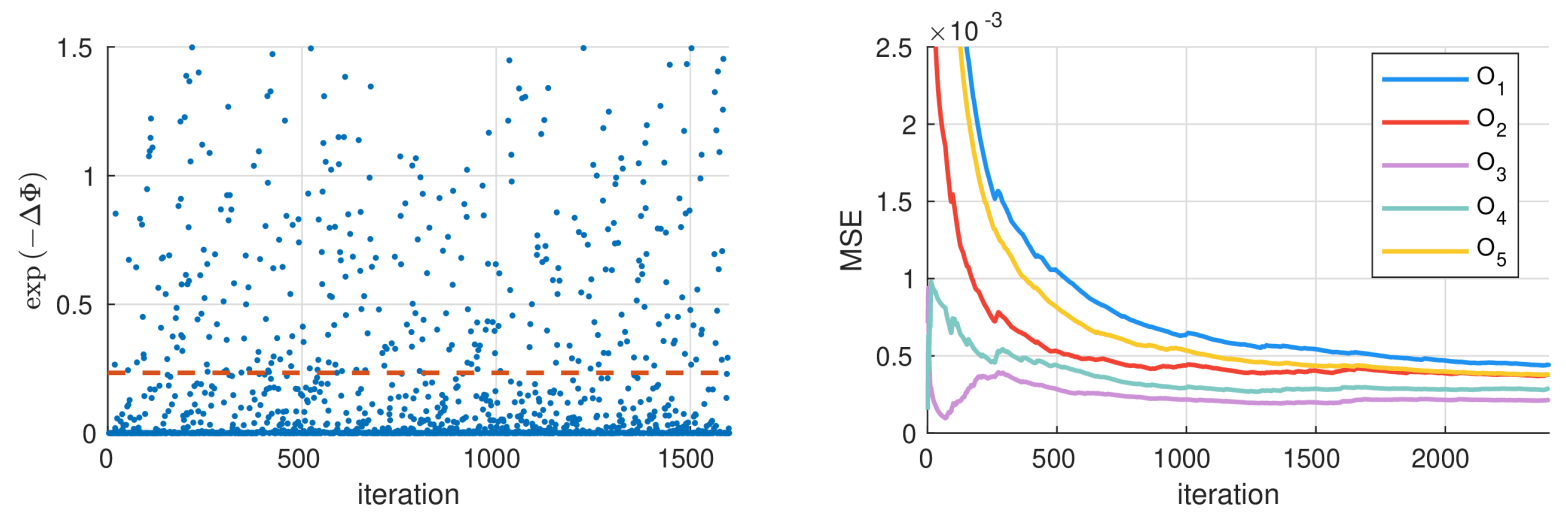

Figure 2: Left figure: transition probability ratio $\exp (-\Delta \Phi)=\exp \left(\Phi^{(k-1)}-\widehat{\Phi}^{(k)}\right)$ is plotted in blue dots and the mean acceptance rate is indicated by the red dashed line. Right figure: mean squared sampling error in test observables. The mean error stabilizes after the 1600th iteration. In both figures, the horizontal axis corresponds to the iteration of the sampling procedure.
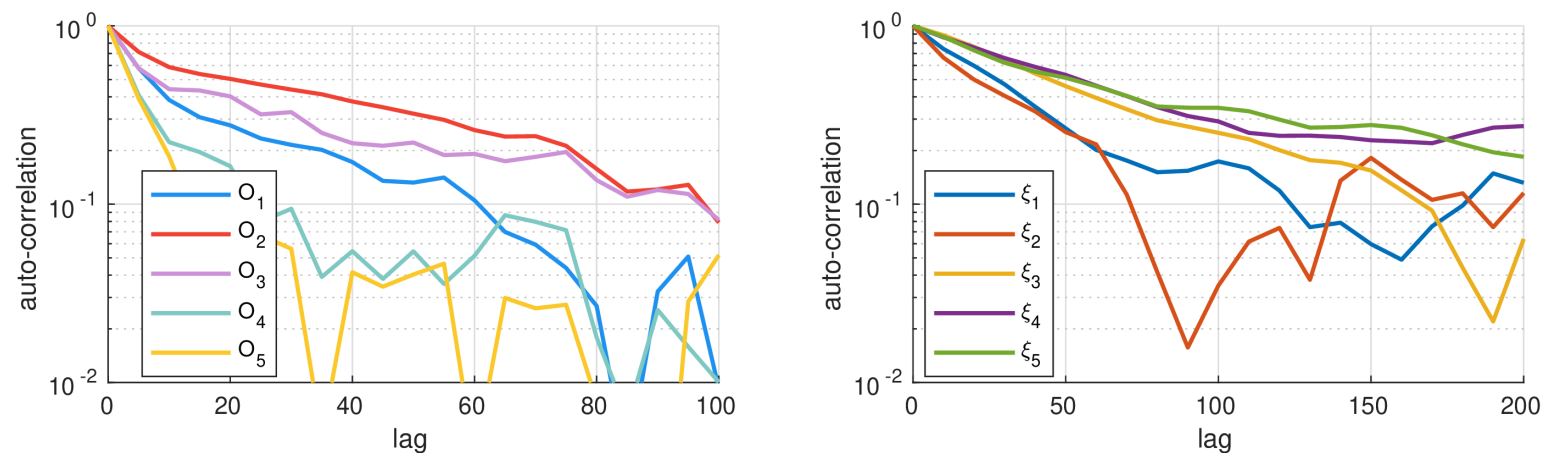

Figure 3: Auto-correlation in test observables (left figure) and in coordinates of the sampled potential (right figure). The horizontal axis corresponds to the lag between samples. When the lag reaches 50, the auto-correlation gets below 0.5 in every variable of both figures.

iterations. We will further discuss the residual between the convergent value and the ground truth in the following sections.

\subsection{Stability result: A numerical proof}

Recall the form of theorem on stability (Cor. 9):

$$
\left|\mathbb{E}_{\pi^{y_{1}}} \bar{O}[\mathfrak{q}]-\mathbb{E}_{\pi^{y_{2}}} \bar{O}[\mathfrak{q}]\right| \lesssim_{r} \| \boldsymbol{y}_{1}-\left.\boldsymbol{y}_{2}\right|_{2}
$$

this inequality describes how the perturbation of output testing observations are bounded by that of the input training observations. To be more specific, we can interpret it in the following two ways:

1. Given noise covariance matrix $\Gamma_{\boldsymbol{\eta}}$, the theorem ensures that the output predictions should rely on the input training observations continuously, thus leading to a stable numerical algorithm.

2. More generally, the theorem also describes how consistent the output predictions on test observ- 

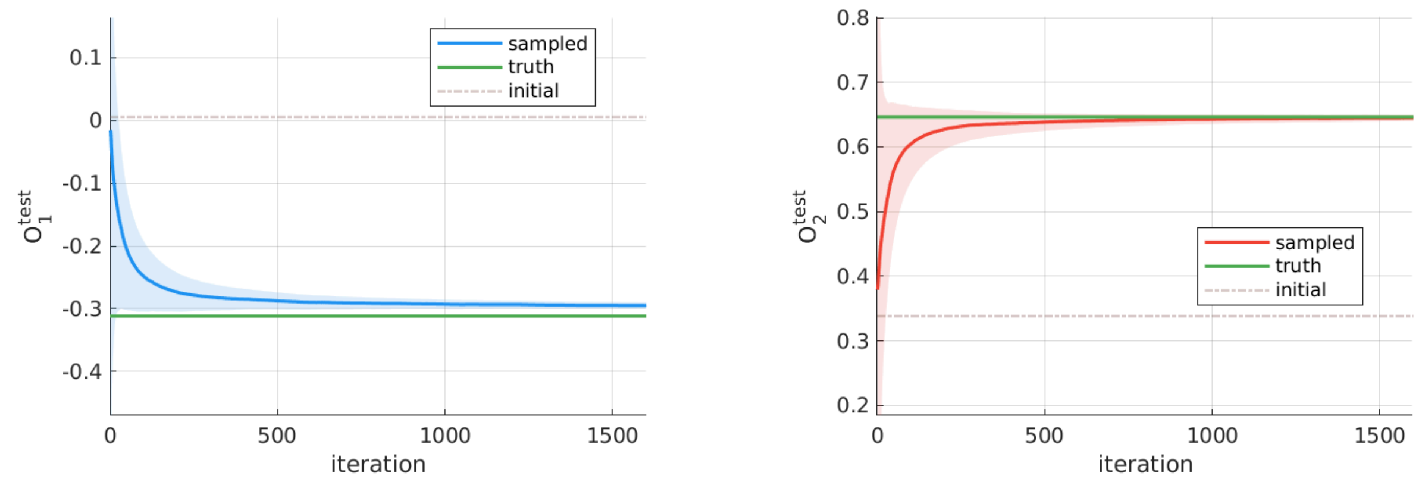

Figure 4: Sampled test observations averaged along the proposal-decision iterations. In each of the two figures, the blue/red line corresponds to the averaged samples for the 1st/2nd test observable. The shaded area indicates twice the standard error with respect to different independent runs. The green line stands for the ground-truth thermal average of the test observable. The faded brown line stands for the value sampled at the initial iteration by setting the potential $V$ to be the harmonic oscillation potential $V_{o}$.
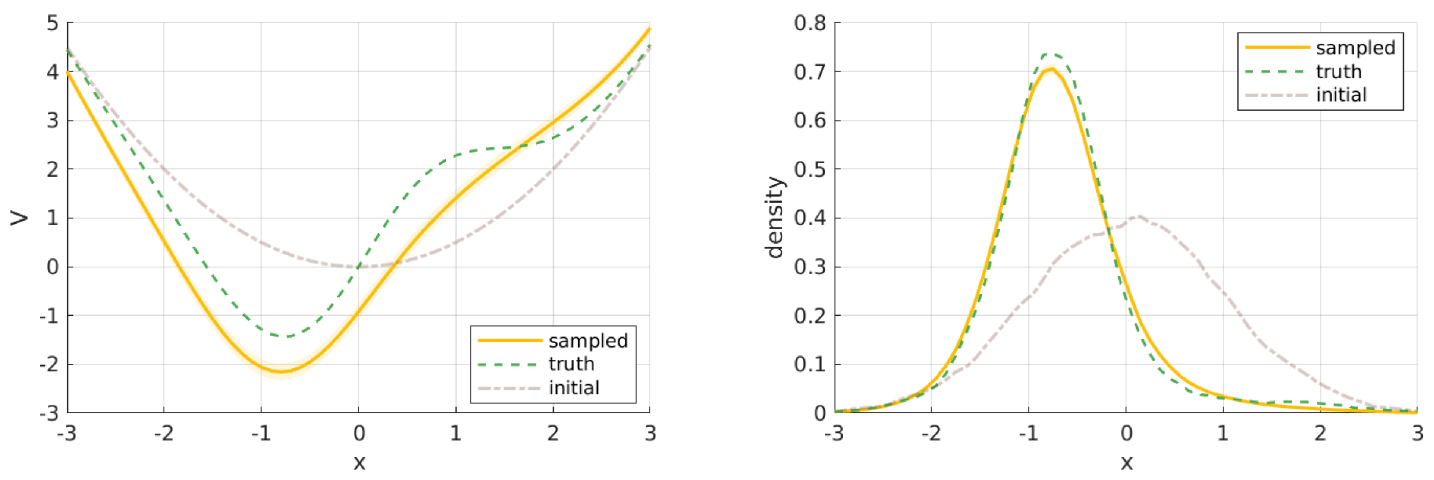

Figure 5: Averaged sampled potential functions $\frac{1}{K_{\text {total }}} \sum_{k=1}^{K_{\text {total }}} V^{(k)}$ (left figure) and density functions (right figure).

Shaded area indicates twice the standard error with respect to different independent runs.

Green dashed line: obtained by setting the potential of the system to the ground-truth potential $V_{\text {truth }}$. Faded brown line: obtained from the harmonic oscillation potential $V_{o}$ (also used as the initial condition $\left.V^{(0)}\right)$.

ables are if the noise covariance is specified. Consider the following formal derivation. Let $\boldsymbol{y}^{*}$ be the thermal average of training observables and $V_{\text {truth }}$ be the potential function. The distribution of the noisy training observation $\boldsymbol{y}$ is $\mathcal{N}\left(\boldsymbol{y}^{*}, \Gamma_{\boldsymbol{\eta}}\right)$, merely a translation of the distribution of the noise $\boldsymbol{\eta}$. According to Jensen's inequality, the expectation of the input disturbance can be estimated by interchanging the expectation operator and the quadratic function:

$$
\mathbb{E}_{\boldsymbol{y} \sim \mathcal{N}\left(\boldsymbol{y}^{*}, \Gamma_{\boldsymbol{\eta}}\right)}\left\|\boldsymbol{y}-\boldsymbol{y}^{*}\right\|_{2} \leq \sqrt{\mathbb{E}_{\mathcal{N}\left(0, \Gamma_{\boldsymbol{\eta}}\right)}\|\boldsymbol{\eta}\|_{2}^{2}}=\sqrt{\operatorname{Tr}\left(\Gamma_{\boldsymbol{\eta}}\right)}
$$


On the other hand, assume that the negative $\log$ likelihood $\Phi(V ; \boldsymbol{y})$ admits a unique minimizer $V_{\text {truth }}$, then as $\operatorname{Tr}\left(\Gamma_{\boldsymbol{\eta}}\right)$ approaches zero, the measure $\mu^{\boldsymbol{y}^{*}}$ induced on the potential space $W^{\mathbf{1}}$ converges to the delta measure $\delta\left(V-V_{\text {truth }}\right)$, in which case the test prediction $\mathbb{E}_{\mathfrak{q} \sim \pi y} \bar{O}[\mathfrak{q}]=$ $\mathbb{E}_{V \sim \mu^{y}} G^{O}(V)$ converges to $G^{O}\left(V_{\text {truth }}\right)$, i.e. the ground-truth thermal average of test observables. To sum up, if we draw a few training observations from $\mathcal{N}\left(\boldsymbol{y}^{*}, \Gamma_{\boldsymbol{\eta}}\right)$ and send them through the inversion process, the average of the predictions on test observables can be written as a double expectation $\mathbb{E}_{\boldsymbol{y} \sim \mathcal{N}\left(\boldsymbol{y}^{*}, \Gamma_{\boldsymbol{\eta}}\right)} \mathbb{E}_{\mathfrak{q} \sim \pi y} \bar{O}[\mathfrak{q}]$. Thus the error between this average and the ground truth can be estimated by the following inequality

$$
\begin{aligned}
\mid \text { error } \mid & =\left|\mathbb{E}_{\boldsymbol{y} \sim \mathcal{N}\left(\boldsymbol{y}^{*}, \Gamma_{\boldsymbol{\eta}}\right)} \mathbb{E}_{\mathfrak{q} \sim \pi^{y}} \bar{O}[\mathfrak{q}]-G^{O}\left(V_{\text {truth }}\right)\right| \\
& \leq\left\{\mathbb{E}_{\boldsymbol{y} \sim \mathcal{N}\left(\boldsymbol{y}^{*}, \Gamma_{\boldsymbol{\eta}}\right)}\left|\mathbb{E}_{\mathfrak{q} \sim \pi^{y}} \bar{O}[\mathfrak{q}]-\mathbb{E}_{\mathfrak{q} \sim \pi y^{*}} \bar{O}[\mathfrak{q}]\right|\right\}+\left|\mathbb{E}_{\mathfrak{q} \sim \pi y^{*}} \bar{O}[\mathfrak{q}]-G^{O}\left(V_{\text {truth }}\right)\right| \\
& \lesssim r\left\{\mathbb{E}_{\boldsymbol{y} \sim \mathcal{N}\left(\boldsymbol{y}^{*}, \Gamma_{\boldsymbol{\eta}}\right)}|| \boldsymbol{y}-\boldsymbol{y}^{*}||_{2}\right\}+\left|\mathbb{E}_{V \sim \mu^{*}} G^{O}(V)-G^{O}\left(V_{\text {truth }}\right)\right| \\
& \lesssim r \sqrt{\operatorname{Tr}\left(\Gamma_{\boldsymbol{\eta}}\right)}+\operatorname{Tr}\left(\Gamma_{\boldsymbol{\eta}}\right) \cdot \sup _{V \in \boldsymbol{W}^{1}} \delta^{2} G^{O}(V) .
\end{aligned}
$$

When the covariance matrix is small enough, we can ignore the second term since it is of higher order than the first one. However, we must point out these derivations are formal since we assume that the negative $\log$ likelihood $\Phi(V ; \boldsymbol{y})$ has only one minimizer $V_{\text {truth }}$ and the second order variational derivative $\delta^{2} G^{O}(V)$ is bounded on $\boldsymbol{W}^{1}$. The conditions are not easy to prove since the first assumption is equivalent to finding a unique $V$ that can reproduce the training observables, which is discussed in Méhats and Pinaud, 2010 with the density function known. The error analysis when multiple minimizers exist is possible, but it is far beyond the scope of the current paper. As is also shown in this section, the algorithm does not rely on the unique minimizer assumption to perform well.

In the following experiments, we will assume different scales for the noise covariance and compare the corresponding test observations. As in the previous section, the noise covariance is set to $\Gamma_{\eta}=$ $\widetilde{\Gamma_{\eta}} I^{N_{1} \times N_{1}}$, where $\widetilde{\Gamma_{\eta}}=0.01,0.03,0.1,0.3$.

Judging from Fig. 6 we can see that a smaller $\widetilde{\Gamma_{\eta}}$ will lead to a more accurate mean and a smaller variance of the sampled test observations. Furthermore, if we fit the error with the scale of noise covariance $\widetilde{\Gamma_{\eta}}=\frac{1}{N_{T}} \operatorname{Tr}\left(\Gamma_{\boldsymbol{\eta}}\right)$ ( $N_{T}$ is the fixed number of training observables), the slope in log scale (see Fig. 7 is around $\frac{1}{2}$, which is consistent with Eqn. 23

\subsection{Numerical study on 2-Level system}

\subsubsection{Problem formulation}

In the previous sections, we have build a rigorous theory on Bayesian inversion in the quantum thermal average problem. It is tempting to extend this theory into a broader area where the quantum system is associated with multiple electronic states and non-adiabatic effects are taken into account. Under a few technical assumptions, the extension can be quite straightforward since the essential procedures are kept the same: the potential function can induce a probability measure on the bead configuration space, while the thermal average can be compared to the ground truth of training observations to 

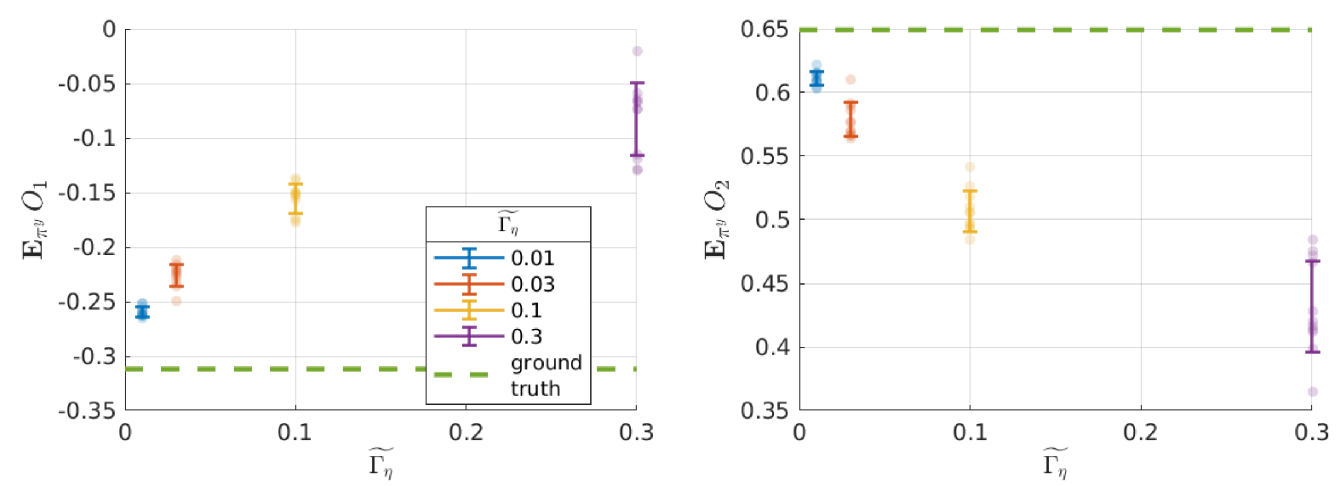

Figure 6: Testing observations compared among experiments if different scales of noise covariance $\widetilde{\Gamma_{\eta}}$ are assumed. Horizontal axis: the scale of noise variance $\widetilde{\Gamma_{\eta}}$. Vertical axis: test observations, where the green dashed line stands for the ground truth. The solid bars stand for the mean and standard error of test observations (faded dots) obtained in numerical experiments.

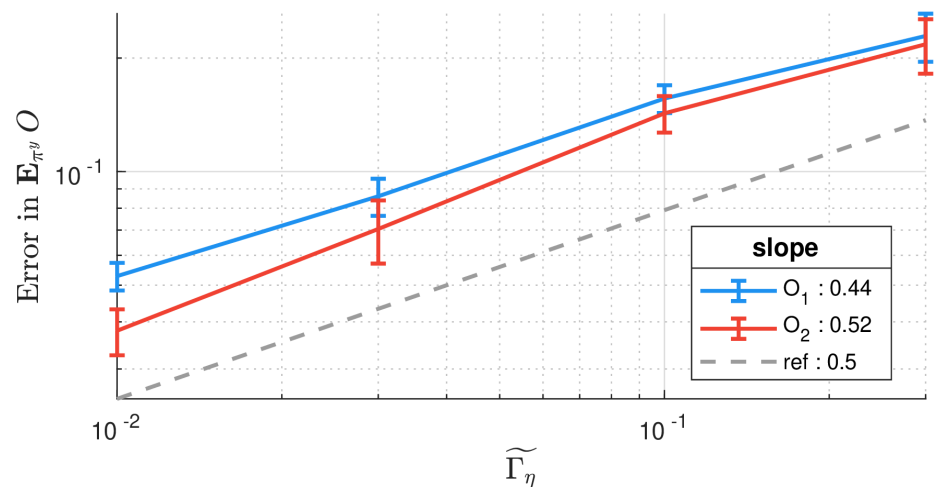

Figure 7: $\log$ scale figure for test observation errors and noise covariance scales $\widetilde{\Gamma_{\eta}}$, indicating that assuming smaller noise covariances can lead to more consistent test observations sampled. Color folded line: averaged test observation errors obtained from numerical experiments. Grey dashed line: reference line with slope $\frac{1}{2}$. The fitted slopes for two folded lines are listed in the legend.

induce a probability measure on the potential function space. The prime difficulty lies in the forward problem of finding an efficient way to sample the distribution on the bead configuration space, which is concerned in [Lu and Zhou, 2017 and PIMD-SH is thus developed. We refer to Sec. B in the Appendix for details. In the following sections we will basically transfer the algorithm proposed for 1-level systems to 2-level systems and demonstrate corresponding numerical studies.

\subsubsection{Notations}

The space of 2-level potential functions $\boldsymbol{V}$ is defined as

$$
W^{2} \triangleq\left\{\boldsymbol{V}=\left(\begin{array}{ll}
V_{00} & V_{01} \\
V_{01} & V_{11}
\end{array}\right): V_{00}, V_{11} \in W^{1}, V_{01} \in W^{g}\right\}
$$


and $W^{g}$ denotes the following mixed-Gaussian-component space

$$
W^{g} \triangleq\left\{V_{01}: V_{01}(x)=\sum_{i=1}^{N_{\text {off }}} A_{i} \exp \left(-\frac{\left(x-c_{i}\right)^{2}}{2 \sigma_{i}^{2}}\right)\right\}
$$

where we have the following assumptions:

- the off-diagonal potential component number $N_{\text {off }}$, location series $\left\{c_{i}\right\}$ and derivation series $\left\{\sigma_{i}\right\}$ are known and fixed,

- each amplitude component $A_{i} \in \mathbb{R}^{+}$is unknown.

\subsubsection{Algorithm explanation}

There are two main differences compared from the 1-level algorithm (Alg. 1):

1. The proposal of $\boldsymbol{V}$ : since we are now dealing with $\boldsymbol{V} \in W^{\mathbf{2}}$, not only the diagonal terms $V_{00}$ and $V_{11}$ are sampled as in the 1-level case, but also the off-diagonal term should be sampled. The prior for $V_{01}$ (essentially for $\left\{A_{i}\right\}$ since the other parameters are fixed) is chosen as the exponential distribution, which can be efficiently proposed by Alg. ??.

2. The sampling of $(\boldsymbol{q}, \boldsymbol{l})$ : it is accomplished by the implementation of PIMD-SH. We refer to Sec. $\mathrm{B}$ in the Appendix for details.

\subsubsection{Numerical study set-up}

Here we conduct tests for the following system:

$$
\begin{aligned}
V_{00} & =\frac{1}{2} x^{2}-\frac{3}{2} \phi_{1}(x) \\
V_{01} & =\exp \left(-2 x^{2}\right) \\
V_{11} & =\frac{1}{2} x^{2}-\frac{3}{4} \phi_{0}(x)-\frac{3}{2} \phi_{1}(x)-\phi_{2}(x)
\end{aligned}
$$

where the off-diagonal potential has $N_{\text {off }}=1$ Gaussian component and $A_{1}=1$ (unknown and to be recovered), $\sigma_{1}=0.5, c_{1}=0$ (fixed and known beforehand). The two diagonal-potentials almost intersect near $x=0$, where $V_{01}$ is significantly positive, providing chances for hopping between layers. Besides, the particle mass $M$ is set to 10, truncation level $L$ to 4 and bead number $N$ to 8 .

The observables we used in training/testing have either diagonal or off-diagonal non-zero entries only. For those only with diagonal entries, we simply set the two diagonal entry to be the same, while those only with off-diagonal entries have the same off-diagonal real-valued entries since the observables are Hermite. The training observables have diagonal Gaussian entries in between $[-2,2]$ 
and off-diagonal Gaussian entries in between $[-1,1]$. The testing observables are

$$
\begin{aligned}
& O_{1}=\left(\begin{array}{cc}
o_{1} & \\
& o_{1}
\end{array}\right) \quad, o_{1}=\exp \left(-\frac{(x-1.25)^{2}}{4}\right) \\
& O_{2}=\left(\begin{array}{cc}
o_{2} & \\
& o_{2}
\end{array}\right) \quad, o_{2}=\exp \left(-\frac{(x+0.25)^{2}}{4}\right)
\end{aligned}
$$

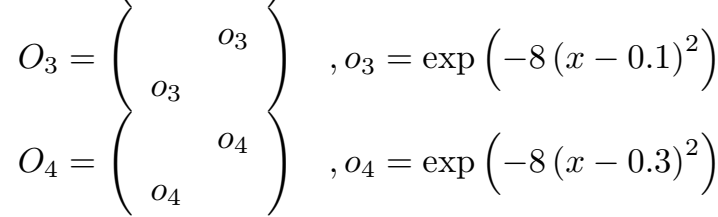

as shown in Fig 8 .
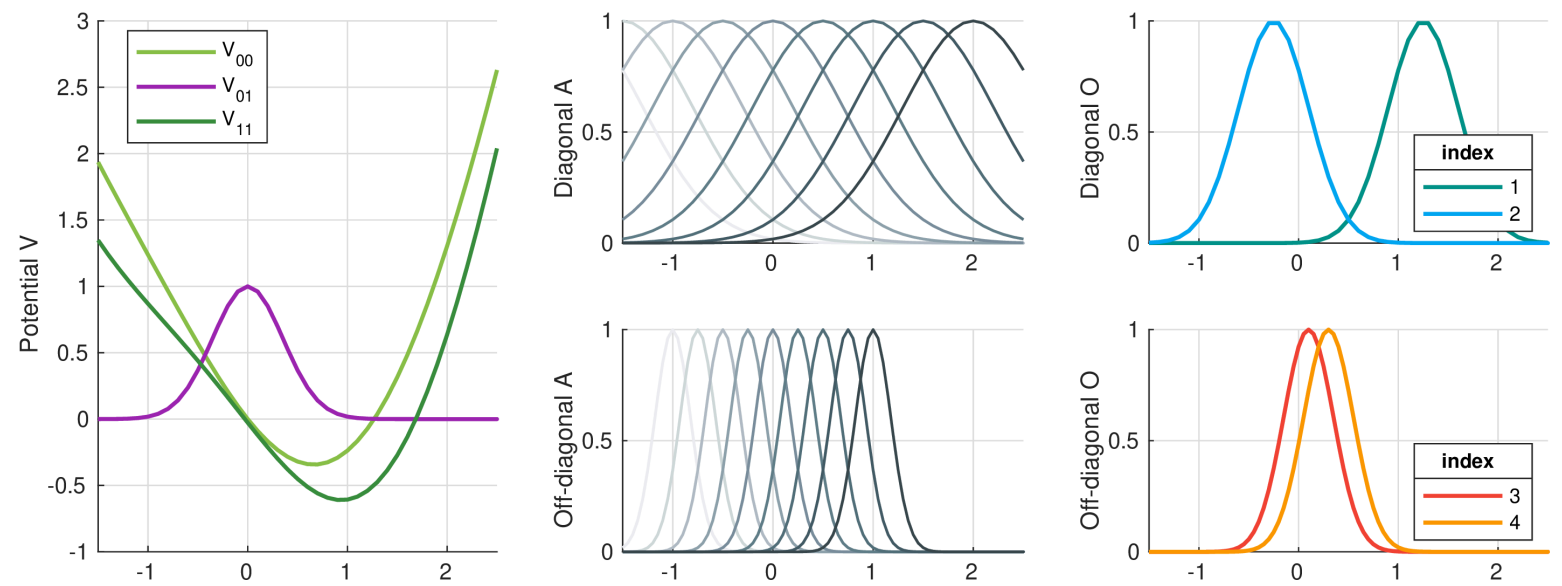

Figure 8: Illustration on ground-truth potential (left-most) and training (middle)/testing (right-most) observables in 2-level system. Top rows show observables with only diagonal entries and bottoms rows show observables with only off-diagonal entries.

\subsubsection{Numerical results and discussions}

Likewise, we demonstrate that the algorithm in the 2-level setting can recover potential landscape and predict test observables. As shown in Fig. 9 and 10 , the sampled average will start from the initial value (marked by the brown line) and converge to the proposed average $\mathbb{E}_{\pi^{y}} O(\mathfrak{q})$, which is quite close to the ground truth (marked by the green line). The potential learned exhibits a tendency to be deeper near $x=1$, which captures the landscape of ground truth potentials.

In a non-adiabatic system, it is quite reasonable to speculate that training with off-diagonal-only observables (i.e. vanish along the diagonal line and only having off-diagonal terms) will help recover the potential landscape more accurately. Fig. 11 shows the comparison between results obtained by training the system with and without off-diagonal-only observables. It is especially clear that the 'with' condition outperforms in the off-diagonal-only test observables in terms of convergence speed and bias. 

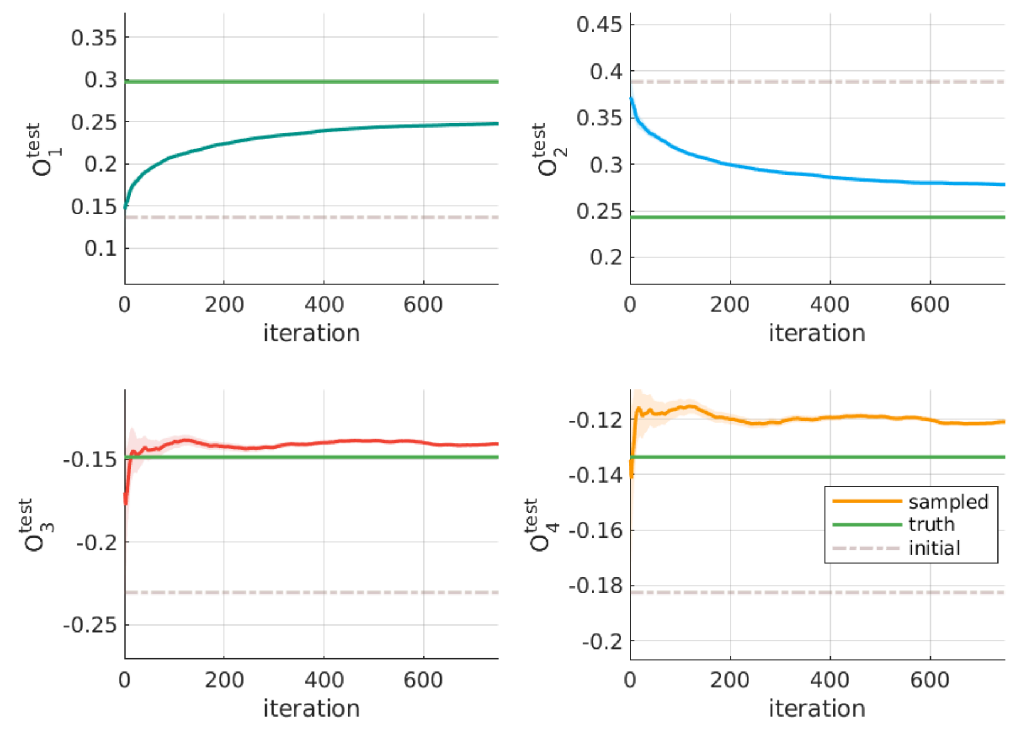

Figure 9: Observations for test observables averaged along the proposal-decision iterations. Top two figures: diagonal-only testing observables $\widehat{O_{1}}$ (teal), $\widehat{O_{2}}$ (light blue). Bottom two figures: off-diagonalonly testing observables $\widehat{O_{3}}$ (red), $\widehat{O_{4}}$ (orange). Green solid line: ground truth. Faded brown dashed line: a guess based on the initial potential function.
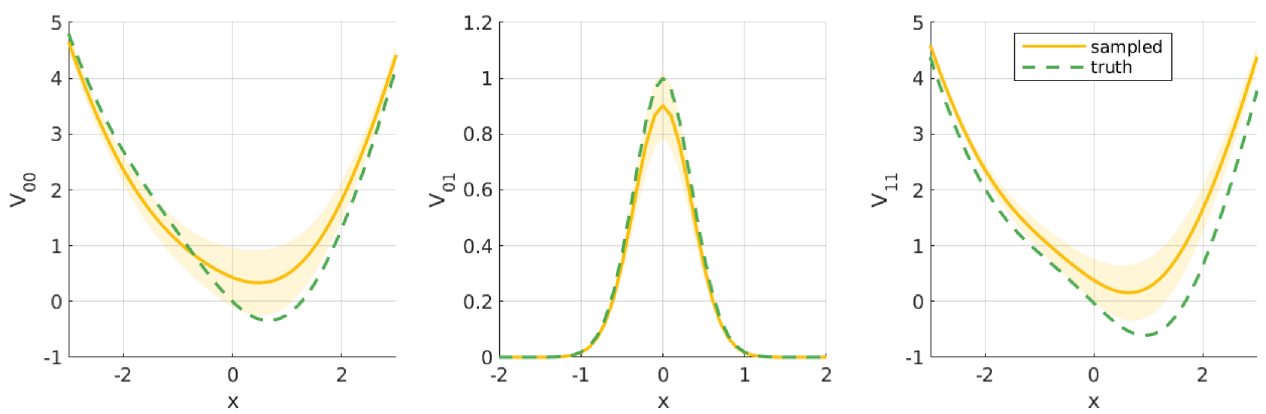

Figure 10: Averaged sampled potentials and densities. The left and right figure demonstrate the diagonal potential landscape on 2 levels, while the middle one demonstrates the off-diagonal potential $V_{01}$. Blue area indicates twice the standard error. Yellow dashed line indicates the ground truth obtained by setting the potential $V$ to be $V_{\text {truth }}$.

\section{Conclusion}

In this paper, we proposed a novel method regarding the inversion problem of quantum thermal average. By adopting the Bayesian inversion framework, we not only obtain a posterior distribution of potential functions based on finite noisy observations, but also the stability result is established under the framework and the numerical algorithm is proved to be efficient. In the numerical section, we have shown that the ensemble average of bounded testing observables can be accurately sampled, and furthermore the error can be controlled by the assumed perturbation in the input training data, 

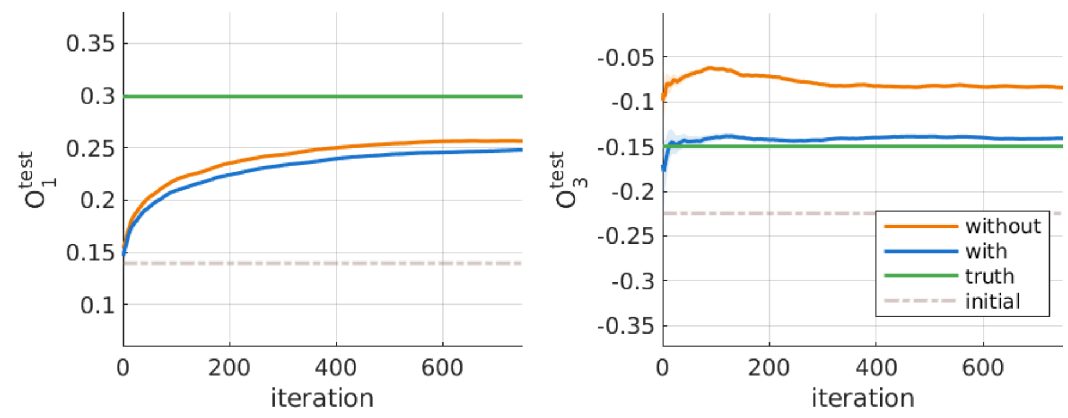

Figure 11: A comparison between training with (blue line) and without (deep orange line) off-diagonalonly observables. The ground truth is marked as the green solid line, and the initial result obtained at the beginning of iterations is marked as the faded brown dashed line. Results on the 2nd and 4th test observables are similar and are not shown in the figure.

which testifies our theoretical result (Thm. 8). We also demonstrated how to apply the algorithm to the non-adiabatic regime. Since the tests in the 2-level setting are preliminary and the accuracy is not satisfactory, it indeed requires more attention and effort on the improvement. One way is to adopt a more efficient sampling algorithm, e.g. the Delayed Rejection Adaptive Metropolis Haario et al., 2006 (with both techniques used together or separately). Another way is to dig deep into the intrinsic mathematical structure of this problem and further improve the precision. In addition, it is not clear yet what observables are favorable in order to have a consistent approximation of the potential function.

Here we ought to mention that this work can easily extend to related problems. First, our method can be extended to systems in higher dimensions. One can easily verify that Thm. 8 still holds if the loop space $X$ is extended to $\mathcal{L} \mathbb{R}^{d}$ and potential space $W^{\mathbf{1}}$ is modified accordingly. As for the numerical implementation, we can expand the potential onto tensor product of Hermite basis. Second, judging from the numerical Sec 6.3, our method fits multi-level systems quite well. A similar theoretical stability result can also be obtained if the Boltzmann distribution follows the form in the single-level system. Future investigations into a more general function space $W^{g}$ setting for the offdiagonal potential functions may yield better results. At the same time, the framework proposed in this work is compatible with all kinds of solvers for the forward problem, so the methods developed in Liu and Liu, 2018, Lu and Zhou, 2018a, Tao et al., 2018 can be seamlessly integrated into the algorithm. Besides, if given multiple training observations, we can also make use of the Ensemble Kalman Filter (EnKF) Mandel, 2009 to improve the accuracy.

\section{References}

[Ananth, 2013] Ananth, N. (2013). Mapping variable ring polymer molecular dynamics: A pathintegral based method for nonadiabatic processes. Journal of Chemical Physics, 139(12):1-8.

[Bui-Thanh et al., 2013] Bui-Thanh, T., Ghattas, O., Martin, J., and Stadler, G. (2013). A Computational Framework for Infinite-Dimensional Bayesian Inverse Problems Part I: The Linearized Case, 
with Application to Global Seismic Inversion. SIAM Journal on Scientific Computing, 35(6):A2494A2523.

[Chayes et al., 1984] Chayes, J. T., Chayes, L., and Lieb, E. H. (1984). The inverse problem in classical statistical mechanics. Communications in Mathematical Physics, 93(1):57-121.

[Cotter et al., 2010] Cotter, S. L., Dashti, M., and Stuart, A. M. (2010). Approximation of Bayesian Inverse Problems for PDEs. SIAM Journal on Numerical Analysis, 48(1):322-345.

[Cotter et al., 2012] Cotter, S. L., Roberts, G. O., Stuart, A. M., and White, D. (2012). MCMC Methods for Functions: Modifying Old Algorithms to Make Them Faster. Statistical Science, 28(3):424446.

[Dashti and Stuart, 2017] Dashti, M. and Stuart, A. M. (2017). The bayesian approach to inverse problems. Handbook of Uncertainty Quantification, pages 311-428.

[Feynman, 1965] Feynman, R. P. (1965). Quantum Mechanics and Path Integrals.

[Haario et al., 2006] Haario, H., Laine, M., Mira, A., and Saksman, E. (2006). DRAM: Efficient adaptive MCMC. Statistics and Computing, 16(4):339-354.

[Habeck, 2014] Habeck, M. (2014). Bayesian approach to inverse statistical mechanics. Physical Review E - Statistical, Nonlinear, and Soft Matter Physics, 89(5):1-7.

[Kleinert, 2009] Kleinert, H. (2009). Path Integrals in Quantum Mechanics, Statistics, Polymer Physics, and Financial Markets. WORLD SCIENTIFIC.

[Leimkuhler and Matthews, 2013a] Leimkuhler, B. and Matthews, C. (2013a). Rational construction of stochastic numerical methods for molecular sampling. Applied Mathematics Research eXpress, 2013(1):34-56.

[Leimkuhler and Matthews, 2013b] Leimkuhler, B. and Matthews, C. (2013b). Robust and efficient configurational molecular sampling via Langevin dynamics. Journal of Chemical Physics, 138(17).

[Lemm, 2000] Lemm, J. (2000). Bayesian approach to inverse time-dependent quantum mechanics. Physics Letters A, 276(1-4):19-24.

[Lemm et al., 2001] Lemm, J., Uhlig, J., and Weiguny, A. (2001). Bayesian reconstruction of approximately periodic potentials for quantum systems at finite temperature. The European Physical Journal B, 20(3):349-366.

[Lemm et al., 2000] Lemm, J. C., Uhlig, J., and Weiguny, A. (2000). Bayesian Approach to Inverse Quantum Statistics. Physical Review Letters, 84(10):2068-2071.

[Lemm et al., 2005] Lemm, J. C., Uhlig, J., and Weiguny, A. (2005). Bayesian approach to inverse quantum statistics: Reconstruction of potentials in the Feynman path integral representation of quantum theory. The European Physical Journal B, 46(1):41-54. 
[Liu et al., 2016] Liu, J., Li, D., and Liu, X. (2016). A simple and accurate algorithm for path integral molecular dynamics with the Langevin thermostat. The Journal of Chemical Physics, 145(2):024103.

[Liu and Liu, 2018] Liu, X. and Liu, J. (2018). Path integral molecular dynamics for exact quantum statistics of multi-electronic-state systems. The Journal of Chemical Physics, 148(10):102319.

[Lu and Zhou, 2017] Lu, J. and Zhou, Z. (2017). Path integral molecular dynamics with surface hopping for thermal equilibrium sampling of nonadiabatic systems. The Journal of Chemical Physics, 146(15):154110.

[Lu and Zhou, 2018a] Lu, J. and Zhou, Z. (2018a). Accelerated sampling by infinite swapping of path integral molecular dynamics with surface hopping. The Journal of Chemical Physics, 148(6):064110.

[Lu and Zhou, 2018b] Lu, J. and Zhou, Z. (2018b). Continuum limit and preconditioned Langevin sampling of the path integral molecular dynamics. pages 1-17.

[Mandel, 2009] Mandel, J. (2009). A Brief Tutorial on the Ensemble Kalman Filter. (January):1-7.

[Méhats and Pinaud, 2010] Méhats, F. and Pinaud, O. (2010). An Inverse Problem in Quantum Statistical Physics. Journal of Statistical Physics, 140(3):565-602.

[Méhats and Pinaud, 2011] Méhats, F. and Pinaud, O. (2011). A problem of moment realizability in quantum statistical physics. Kinetic and Related Models, 4(4):1143-1158.

[Menzeleev et al., 2014] Menzeleev, A. R., Bell, F., and Miller, T. F. (2014). Kinetically constrained ring-polymer molecular dynamics for non-adiabatic chemical reactions. The Journal of Chemical Physics, 140(6):064103.

[Navrotskaya, 2014] Navrotskaya, I. (2014). The Classical Inverse Problem for Multi-Particle Densities in the Canonical Ensemble Formulation. pages 1-12.

[Nguyen et al., 2017] Nguyen, H. C., Zecchina, R., and Berg, J. (2017). Inverse statistical problems: from the inverse Ising problem to data science. Advances in Physics, 66(3):197-261.

[Petra et al., 2014] Petra, N., Martin, J., Stadler, G., and Ghattas, O. (2014). A Computational Framework for Infinite-Dimensional Bayesian Inverse Problems, Part II: Stochastic Newton MCMC with Application to Ice Sheet Flow Inverse Problems. SIAM Journal on Scientific Computing, 36(4):A1525-A1555.

[Stock and Thoss, 2005] Stock, G. and Thoss, M. (2005). Classical Description of Nonadiabatic Quantum Dynamics. In Adv. Chem. Phys., volume 131, pages 243-375.

[Stuart, 2010] Stuart, A. M. (2010). Inverse problems: A Bayesian perspective. Acta Numerica, 19(May 2010):451-559.

[Tao et al., 2018] Tao, X., Shushkov, P., and Miller, T. F. (2018). Path-integral isomorphic Hamiltonian for including nuclear quantum effects in non-adiabatic dynamics. The Journal of Chemical Physics, 148(10):102327. 
[Zhang et al., 2017] Zhang, Z., Liu, X., Chen, Z., Zheng, H., Yan, K., and Liu, J. (2017). A unified thermostat scheme for efficient configurational sampling for classical/quantum canonical ensembles via molecular dynamics. The Journal of Chemical Physics, 147(3):034109.

\section{ACKNOWLEDGMENTS}

Z. Zhou was supported by NSFC grant No. 11801016. Z. Zhou would like to thank Jianfeng Lu and Thomas F. Miller III for helpful discussions. Z. Chen would like to thank Tiejun Li for guidance in Chen's undergraduate study.

\section{A Analysis Supplements}

Lemma 12. The vector space $W^{\mathbf{1}} \triangleq L^{2}(\mathbb{R}) \cap L^{\infty}(\mathbb{R})$ is a Banach space under the norm

$$
\|f\|_{W^{1}} \triangleq\|f\|_{L^{2}(\mathbb{R})}+\|f\|_{L^{\infty}(\mathbb{R})} .
$$

Proof. It suffices to show that a Cauchy sequence $\left\{f_{n}\right\}$ in $W$ converges in $W$. In fact, $\left\{f_{n}\right\}$ is a Cauchy sequence both in $L^{2}(\mathbb{R})$ and in $L^{\infty}(\mathbb{R})$. Denote the corresponding limit as $f_{n} \stackrel{L^{2}(\mathbb{R})}{\longrightarrow} h$ and $f_{n} \stackrel{L^{\infty}(\mathbb{R})}{\longrightarrow} \widetilde{h}$.

Restricted on a given interval $[a, b]$, notice that $\|\cdot\|_{L^{2}([a, b])} \leq\|\cdot\|_{L^{2}(\mathbb{R})}$ and $\|\cdot\|_{L^{\infty}([a, b])} \leq\|\cdot\|_{L^{\infty}(\mathbb{R})}$, we have $h \in L^{2}([a, b])$ and $\widetilde{h} \in L^{\infty}([a, b])$. Notice that for $g_{n} \triangleq f_{n}-\widetilde{h} \in L^{\infty}([a, b])$,

$$
\left\|g_{n}\right\|_{L^{2}([a, b])} \leq \sqrt{(b-a)\left\|g_{n}\right\|_{L^{\infty}([a, b])}^{2}} \leq \sqrt{b-a}\left\|g_{n}\right\|_{L^{\infty}([a, b])},
$$

so $g_{n}$ converges to 0 in $L^{2}([a, b])$, i.e. $f_{n} \stackrel{L^{2}([a, b])}{\longrightarrow} \widetilde{h}$. But $f_{n}$ also converges to $h$ in $L^{2}([a, b])$, so $h$ and $\widetilde{h}$ a.e. agree with each other on $[a, b]$.

Then, since $h$ and $\widehat{h}$ a.e. agree on $[-N, N]$, they a.e. agree on $\mathbb{R}$, so $f_{n}$ converges to $h \in$ $L^{2}(\mathbb{R}) \cap L^{\infty}(\mathbb{R})$ under the $\|\cdot\|_{W^{1}}$ norm.

Definition 13. The Hermite function sequence $\left\{\phi_{n}\right\}$ is defined as

$$
\phi_{n}(x) \triangleq(-1)^{n} \cdot\left(2^{n} n ! \sqrt{\pi}\right)^{-\frac{1}{2}} \cdot e^{\frac{x^{2}}{2}} \cdot \frac{d^{n}}{d x^{n}} e^{-x^{2}} .
$$

Theorem 14. Let $D \subset \mathbb{R}^{d}$ be a compact domain, $\left\{\gamma_{k}\right\}_{k \geq 0}$ be a real-valued sequence and $\left\{\xi_{k}\right\}_{k \geq 0}$ be countably many centered i.i.d. random variables with bounded moments of all orders. Assume that $\left\{\psi_{k}\right\}_{k \geq 0}$ is a sequence of Hölder functions satisfying

$$
\begin{gathered}
\left|\psi_{k}(x)\right| \leq C_{1}, \quad \forall k \geq 0, x \in D \\
\left|\psi_{k}(x)-\psi_{k}(y)\right| \leq C_{2} k^{t}|x-y|^{\alpha}, \quad \forall k \geq 0, x \in D
\end{gathered}
$$


where $C_{1}, C_{2}$ are constants and $\alpha \in(0,1], t>0$. Suppose there is some $\delta \in(0,2)$ such that

$$
S_{1} \triangleq \sum_{k \geq 0} \gamma_{k}^{2}<\infty \text { and } S_{2} \triangleq \sum_{k \geq 0} \gamma_{k}^{2-\delta} k^{t \delta}<\infty
$$

Then $u$ defined by

$$
u \triangleq \sum_{k \geq 0} \gamma_{k} \xi_{k} \psi_{k}
$$

is a.s. finite for every $x \in D$, and $u \in C^{0, \alpha^{\prime}}(D)$ for every $\alpha^{\prime}<\alpha \delta / 2$ (i.e. u is Hölder continuous for every exponent smaller than $\alpha \delta / 2$ ).

This theorem is a direct corollary which can be found in Dashti and Stuart, 2017, Page 91.

Proposition 15. Assume the positive sequence $\gamma_{j}=\mathcal{O}\left(j^{-s}\right)$ with $s>1$ and $\left\{\xi_{j}\right\}_{j=1}^{\infty}$ are i.i.d. random variables with finite second moment. Denote the Hermite functions as $\left\{\phi_{n}\right\}$ defined in 13 . Then the function series $\sum_{j=0}^{\infty} \xi_{j} \gamma_{j} \phi_{j} \mathbb{P}$-a.s. converges in $W^{\mathbf{1}}=L^{\infty} \cap L^{2}$ with norm $\|\cdot\|_{W^{1}}=\|\cdot\|_{L^{2}}+\|\cdot\|_{L^{\infty}}$.

Proof. We will use the following lemma without proof: let $\left\{I_{j}\right\}_{j=0}^{\infty}$ be a sequence of $\mathbb{R}^{+}$-valued independent random variables, then the following equivalence holds:

$$
\sum_{j=0}^{\infty} I_{j}<+\infty \mathbb{P} \text {-a.s. } \Longleftrightarrow \sum_{j=0}^{\infty} \mathbb{E}\left(I_{j} \wedge 1\right)<\infty .
$$

For the $L^{\infty}$ part, we denote $I_{j}^{(1)}=\left\|\xi_{j} \gamma_{j} \phi_{j}\right\|_{L^{\infty}}$. By Cramér's inequality $\left\|\phi_{n}\right\|_{L^{\infty}} \leq \pi^{-\frac{1}{4}}$, we have

$$
\sum_{j=0}^{\infty} \mathbb{E} I_{j}^{(1)}=\sum_{j=0}^{\infty} \gamma_{j}\left\|\phi_{j}\right\|_{L^{\infty}} \mathbb{E}\left|\xi_{j}\right| \leq \pi^{-\frac{1}{4}} \mathbb{E}\left|\xi_{j}\right| \sum_{j=0}^{\infty} \gamma_{j}
$$

For the $L^{2}$ part, we denote $I_{j}^{(2)}=\left\|\xi_{j} \gamma_{j} \phi_{j}\right\|_{L^{2}}$, then

$$
\sum_{j=0}^{\infty} \mathbb{E} I_{j}^{(2)}=\mathbb{E}\left|\xi_{j}\right| \sum_{j=0}^{\infty} \gamma_{j}
$$

Since we already assumed that $\gamma_{j}=\mathcal{O}\left(j^{-s}\right)$ with $s>1$, the infinite sums $\sum_{j=0}^{\infty} \gamma_{j}$ and $\sum_{j=0}^{\infty} \gamma_{j}^{2}$ are finite, so

$$
\begin{aligned}
\sum_{j=0}^{\infty} \mathbb{E}\left(\left\|\xi_{j} \gamma_{j} \phi_{j}\right\|_{W^{1}} \wedge 1\right) & \leq \sum_{j=0}^{\infty} \mathbb{E} I_{j}^{(1)}+\sum_{j=0}^{\infty} \mathbb{E} I_{j}^{(2)} \\
& \leq\left(1+\pi^{-\frac{1}{4}}\right) \mathbb{E}\left|\xi_{j}\right| \sum_{j=0}^{\infty} \gamma_{j} \\
& <\infty
\end{aligned}
$$

According to the lemma we stated at the beginning of the proof, the infinite sum $\sum_{i=0}^{\infty}\left\|\xi_{j} \gamma_{j} \phi_{j}\right\|_{W^{1}}$ $\mathbb{P}$-a.s. converges. By the completeness of $W^{\mathbf{1}}$, the function series $\sum_{i=0}^{\infty} \xi_{i} \gamma_{i} \phi_{i} \mathbb{P}$-a.s. converges to a $W^{1}$ function. 
Proposition 16. Assume the positive sequence $\gamma_{j}=\mathcal{O}\left(j^{-s}\right)$ with $s>\frac{1}{2}$ and $\xi_{j} \stackrel{i . i . d .}{\sim} \mathcal{N}(0,1)$. Denote the Hermite functions defined in 13 as $\left\{\phi_{n}\right\}$. Then for all $\beta<\frac{4 s-2}{4 s+1}$, the function series $\sum_{j=0}^{\infty} \xi_{j} \gamma_{j} \phi_{j}$ $\mathbb{P}$-a.s. converges in $C^{0, \beta}(\mathbb{R})$.

Proof. First we fix the domain $D_{R}$ restricted to $[-R, R]$. By Thm. 14, the series of interest has proper regularity as long as $S_{1}$ and $S_{2}$ are finite. The key of this proof is to identify constants $t$ and $\alpha$ satisfying

$$
\left|\phi_{n}(x)-\phi_{n}(y)\right| \leq C_{2} n^{t}|x-y|^{\alpha}
$$

for any $x, y \in D_{R}$ and $n \in \mathbb{N}$.

For Hermite basis functions, $\arg \max \left|\phi_{n}^{\prime}\right|=0$ if $n$ is odd. If we take $p=\frac{n+1}{2}$, we can have the following equation after some tedious calculations:

$$
\left|\phi_{n}^{\prime}(0)\right|=\frac{\sqrt{(2 p-1) !}}{2^{p-1}(2 \pi)^{\frac{1}{4}}(p-1) !} .
$$

By Stirling formula,

$$
\log \left|\phi_{n}^{\prime}(0)\right|=\frac{1}{4} \log (n+1)-\frac{1}{2} \log \pi-\frac{1}{8(n+1)}+\mathcal{O}\left(n^{-2}\right),
$$

which indicates that $t=\frac{1}{4}$.

By assumption, $\gamma_{j}=\mathcal{O}\left(j^{-s}\right)$, we have

$$
\begin{gathered}
S_{1}<\infty \Longleftrightarrow s>\frac{1}{2}, \\
S_{2}<\infty \Longleftrightarrow-(2-\delta) s+t \delta<-1 \Longleftrightarrow \delta<\frac{2 s-1}{s+t}=\frac{8 s-4}{4 s+1} .
\end{gathered}
$$

So by Thm. 14, the function series $\sum_{j=0}^{\infty} \xi_{j} \gamma_{j} \phi_{j}$ is Hölder continuous on $D_{R}$ for every exponent smaller than $\frac{4 s-2}{4 s+1}$. Since $\mathbb{R}=\bigcup_{R=1}^{\infty} D_{R}$ and the Hölder continuity of the function series $\mathbb{P}$-a.s. holds for countably many $R$, it also $\mathbb{P}$-a.s. holds on $\mathbb{R}$.

\section{B RPR in Two-level Systems}

\section{B.1 Ring polymer representation}

Without loss of generality, a few assumptions have been made in Lu and Zhou, 2017:

1. The total level of energy in the system is 2 .

2. The potential function $V=\left(\begin{array}{cc}V_{00} & V_{01} \\ V_{01} & V_{11}\end{array}\right)$ is Hermite and the off-diagonal term $V_{01}$ keeps its sign.

3. The observable $\widehat{A}$ only depends on position $q$. 
To fully describe the bead, a level index vector $\boldsymbol{l} \in\{0,1\}^{N}$ is needed besides the position configuration $\boldsymbol{q} \in \mathbb{R}^{N}$. By a similar induction and introduction of auxiliary momentum variable $\boldsymbol{p}$ as in the single level system, we arrive at the following formula for the thermal average

$$
\langle\widehat{A}\rangle=\frac{1}{\mathcal{Z}_{N}} \int_{\mathbb{R}^{N}} \int_{\mathbb{R}^{N}} \sum_{\boldsymbol{l} \in\{0,1\}^{N}} W_{N}[A] e^{-\beta_{N} H_{N}(\boldsymbol{q}, \boldsymbol{p}, \boldsymbol{l})} d \boldsymbol{q} d \boldsymbol{p}+\mathcal{O}\left(N^{-2}\right),
$$

where the weight function is given by

$W_{N}[A](\boldsymbol{q}, \boldsymbol{p}, \boldsymbol{l}) \triangleq \frac{1}{N} \sum_{k=1}^{N}\left\{\left\langle l_{k}\left|A\left(q_{k}\right)\right| l_{k}\right\rangle-\boldsymbol{s g n}\left(V_{l_{k} \overline{l_{k}}}\right)\left\langle l_{k}\left|A\left(q_{k}\right)\right| \overline{l_{k}}\right\rangle \exp \left[\beta_{N}\left(\left\langle l_{k}\left|G_{k}\right| l_{k+1}\right\rangle-\left\langle\overline{l_{k}}\left|G_{k}\right| l_{k+1}\right\rangle\right)\right]\right\}$,

the Hamiltonian given by

$$
H_{N}(\boldsymbol{q}, \boldsymbol{p}, \boldsymbol{l})=\sum_{k=1}^{N}\left\langle l_{k}\left|G_{k}\right| l_{k+1}\right\rangle
$$

the matrix $G_{k}$ given by

$$
\left\langle l\left|G_{k}\right| l^{\prime}\right\rangle=\left\{\begin{array}{ll}
\frac{p_{k}^{2}}{2 M}+\frac{M\left(q_{k}-q_{k+1}\right)^{2}}{2 \beta_{N}^{2}}+V_{l l}\left(q_{k}\right)-\frac{1}{\beta_{N}} \log \left(\cosh \left(\beta_{N}\left|V_{01}\left(q_{k}\right)\right|\right)\right) & l=l^{\prime} \\
\frac{p_{k}^{2}}{2 M}+\frac{M\left(q_{k}-q_{k+1}\right)^{2}}{2 \beta_{N}^{2}}+\frac{V_{00}\left(q_{k}\right)+V_{11}\left(q_{k}\right)}{2}-\frac{1}{\beta_{N}} \log \left(\sinh \left(\beta_{N}\left|V_{01}\left(q_{k}\right)\right|\right)\right) & l \neq l^{\prime}
\end{array},\right.
$$

shorthand notation $\overline{l_{i}}=1-l_{i}$ and $\mathcal{Z}_{N} \triangleq \int_{\mathbb{R}^{N}} \int_{\mathbb{R}^{N}} \sum_{\boldsymbol{l} \in\{0,1\}^{N}} \exp \left[-\beta_{N} H_{N}(\boldsymbol{q}, \boldsymbol{p}, \boldsymbol{l})\right] d \boldsymbol{q} d \boldsymbol{p}$ the normalization constant. We leave the detailed derivations and discussions to Lu and Zhou, 2017.

\section{B.2 PIMD-SH: An abstract}

The central idea of PIMD-SH is to construct a reversible Markov process that the invariant distribution

is exactly $\frac{1}{\mathcal{Z}_{N}} \exp \left(-\beta_{N} H_{N}(\boldsymbol{q}, \boldsymbol{p}, \boldsymbol{l})\right)$. To achieve this, the dynamics of the position and momentum variable $\boldsymbol{z} \triangleq(\boldsymbol{q}, \boldsymbol{p})$ is similar to Eqn. 9 as

$$
\left\{\begin{array}{l}
d \boldsymbol{q}=\nabla_{\boldsymbol{p}} H_{N}(\boldsymbol{q}(t), \boldsymbol{p}(t), \boldsymbol{l}(t)) d t \\
d \boldsymbol{p}=-\nabla_{\boldsymbol{q}} H_{N}(\boldsymbol{q}(t), \boldsymbol{p}(t), \boldsymbol{l}(t)) d t-\gamma \boldsymbol{p} d t+\sqrt{\frac{2 \gamma M}{\beta_{N}}} d \boldsymbol{B}
\end{array} .\right.
$$

The evolution of $\boldsymbol{l}(t)$ follows a surface hopping type dynamics, essentially a Q-process: for given index configuration $\boldsymbol{l}$, we restrict that the post-changing state can only be $\overline{\boldsymbol{l}}$ or those $\boldsymbol{l}^{\prime}$ which has distance 1 to $\boldsymbol{l}$. The hopping intensity from $\boldsymbol{l}$ to other possible states is determined by the following detailed balance condition

$$
p_{\boldsymbol{l}^{\prime}, \boldsymbol{l}}(\boldsymbol{z}) \exp \left[-\beta_{N} H_{N}(\boldsymbol{z}, \boldsymbol{l})\right]=p_{\boldsymbol{l}, \boldsymbol{l}^{\prime}}(\boldsymbol{z}) \exp \left[-\beta_{N} H_{N}\left(\boldsymbol{z}, \boldsymbol{l}^{\prime}\right)\right]
$$

up to an overall scaling parameter $\boldsymbol{\eta}$.

In Lu and Zhou, 2017 the choice for $p_{\boldsymbol{l}^{\prime}, \boldsymbol{l}}(\boldsymbol{z})$ was $\exp \left[\frac{\beta_{N}}{2}\left(H_{N}(\boldsymbol{z}, \boldsymbol{l})-H_{N}\left(\boldsymbol{z}, \boldsymbol{l}^{\prime}\right)\right)\right]$, which is not upper-bounded, causing numerical difficulties if the overall hopping probability exceeds 1 . Hence, in this article we propose the Hasting-style transition intensity $\min \left\{1, \exp \left[\frac{\beta_{N}}{2}\left(H_{N}(\boldsymbol{z}, \boldsymbol{l})-H_{N}\left(\boldsymbol{z}, \boldsymbol{l}^{\prime}\right)\right)\right]\right\}$. 
Once the theory for dynamics is fully established, we can apply the theorem of ergodicity

$$
\langle\widehat{A}\rangle=\lim _{T \rightarrow \infty} \frac{1}{T} \int_{0}^{T} W_{N}[A](\widetilde{z}(t)) d t
$$

and calculate the thermal average by truncating the formula above with a finite time span.

\section{Bayesian Inversion Supplements}

\section{C.1 Distance between measures}

Definition 17. The total variation distance between two measures $\mu$ and $\mu^{\prime}$ is

$$
d_{T V}\left(\mu, \mu^{\prime}\right) \triangleq \frac{1}{2} \int\left|\frac{d \mu}{d \nu}-\frac{d \mu^{\prime}}{d \nu}\right| d \nu
$$

Definition 18. The Hellinger distance between $\mu$ and $\mu^{\prime}$ is

$$
d_{H e l l}\left(\mu, \mu^{\prime}\right) \triangleq \sqrt{\frac{1}{2} \int\left(\sqrt{\frac{d \mu}{d \nu}}-\sqrt{\frac{d \mu^{\prime}}{d \nu}}\right)^{2} d \nu}
$$

Lemma 19. The total variation metric and the Hellinger metric are related by the following inequalities

$$
\frac{1}{\sqrt{2}} d_{T V}\left(\mu, \mu^{\prime}\right) \leq d_{H e l l}\left(\mu, \mu^{\prime}\right) \leq d_{T V}\left(\mu, \mu^{\prime}\right)^{\frac{1}{2}}
$$

\section{C.2 Theorem on conditional variables}

Theorem 20. Let $(\mathcal{X}, A)$ and $(\mathcal{Y}, B)$ denote a pair of measurable spaces and let $\nu$ and $\pi$ be probability measures on $\mathcal{X} \times \mathcal{Y}$. We assume that $\nu \ll \pi$. Thus there exists $\pi$-measurable $\phi: \mathcal{X} \times \mathcal{Y} \rightarrow \mathbb{R}$ with $\phi \in L_{\pi}^{1}$ and

$$
\frac{d \nu}{d \pi}(x, y)=\phi(x, y) .
$$

Assume that the conditional random variable $x \mid y$ exists under $\pi$ with probability distribution denoted by $\pi^{y}(d x)$. Then the conditional random variable $x \mid y$ exists under $\nu$, with probability distribution denoted by $\nu^{y}(d x)$. Furthermore, $\nu^{y} \ll \pi^{y}$ and if $c(y) \triangleq \int_{X} \phi(x, y) d \pi^{y}(x)>0$, then

$$
\frac{d \nu^{y}}{d \pi^{y}}(x)=\frac{1}{c(y)} \phi(x, y) \text {. }
$$

The theorem can be found in Dashti and Stuart, 2017, Page 28. 


\section{C.3 Well-posedness result}

Notation. In this section, let $\mathcal{X}$ and $\mathcal{Y}$ be two separable Banach spaces, and $\mu_{0}$ a measure on $\mathcal{X}$. Given $y \in \mathcal{Y}, \mu^{y}$ is defined as the measure absolutely continuous to $\mu_{0}$ by

$$
\begin{aligned}
\frac{d \mu^{y}}{d \mu_{0}}(x) & =\frac{1}{Z(y)} \exp (-\Phi(x ; y)) \\
Z(y) & =\int_{\mathcal{X}} \exp (-\Phi(x ; y)) \mu_{0}(d x) .
\end{aligned}
$$

Assumption 21. Let $\mathcal{X}^{\prime}$ is a separable subspace of $\mathcal{X}$ and assume that $\Phi \in C\left(\mathcal{X}^{\prime} \times \mathcal{Y} ; \mathbb{R}\right)$. Assume further that there are functions $M_{i}: \mathbb{R}^{+} \times \mathbb{R}^{+} \rightarrow \mathbb{R}^{+}, i=1,2$, monotonic non-decreasing separately in each argument, and with $M_{2}$ strictly positive, such that for all $u \in \mathcal{X}^{\prime}, y, y_{1}, y_{2} \in B \mathcal{Y}(0, r)$,

$$
\begin{gathered}
\Phi(u ; y) \geq-M_{1}\left(r,\|u\|_{\mathcal{X}}\right), \\
\left|\Phi\left(u ; y_{1}\right)-\Phi\left(u ; y_{2}\right)\right| \leq M_{2}\left(r,\|u\|_{\mathcal{X}}\right)\left\|y_{1}-y_{2}\right\|_{\mathcal{Y}} .
\end{gathered}
$$

Theorem 22. Assume that Assu. 21 holds. Assume that the prior measure is a.s. restricted on the separable subspace $\mathcal{X}^{\prime}$ (i.e. $\mu_{0}\left(\mathcal{X}^{\prime}\right)=1$ ) and that $\mu_{0}\left(\mathcal{X}^{\prime} \cap S\right)>0$ for some bounded set $S$ in $\mathcal{X}$. Assume additionally that, for every fixed $r>0$,

$$
\exp \left(M_{1}\left(r,\|u\|_{\mathcal{X}}\right)\right)\left(1+M_{2}\left(r,\|u\|_{\mathcal{X}}\right)^{2}\right) \in L_{\mu_{0}}^{1}(\mathcal{X} ; \mathbb{R}) .
$$

Then we have the following asymptotic inequality

$$
d_{\text {Hell }}\left(\mu^{y}, \mu^{y^{\prime}}\right) \lesssim_{r}\left\|y-y^{\prime}\right\|_{\mathcal{Y}} .
$$

The proof can be found in Dashti and Stuart, 2017], Page 38-39. Thm. 22 ensures that the distance between induced posterior probability measures is bounded by the distance between different dependent variables.

Lemma 23. Given a function $f \in L_{\mu^{y}}^{\infty}(X ; \mathbb{R}) \cap L_{\mu^{y^{\prime}}}^{\infty}(X ; \mathbb{R})$, we have

$$
\left|\mathbb{E}^{\mu^{y}} f(u)-\mathbb{E}^{\mu^{y^{\prime}}} f(u)\right| \leq C(f) d_{T V}\left(\mu^{y}, \mu^{y^{\prime}}\right),
$$

which leads to

$$
\left|\mathbb{E}^{\mu^{y}} f(u)-\mathbb{E}^{\mu^{y^{\prime}}} f(u)\right| \lesssim_{r}\left\|y-y^{\prime}\right\|_{\mathcal{Y}}
$$

for fixed $f$.

Proof. By the definition of $d_{T V}$, there exists a measure $\nu$ s.t. both $\mu^{y}$ and $\mu^{y^{\prime}}$ are absolutely continuous 
w.r.t. $\nu$; for example, take $\nu=\frac{1}{2}\left(\mu^{y}+\mu^{y^{\prime}}\right)$. Then a direct calculation shows that

$$
\begin{aligned}
\left|\mathbb{E}^{\mu^{y}} f(u)-\mathbb{E}^{\mu^{y^{\prime}}} f(u)\right| & =\left|\int_{X} f d \mu^{y}(u)-\int_{X} f d \mu^{y^{\prime}}(u)\right| \\
& =\left|\int_{X} f \frac{d \mu^{y}}{d \nu} d \nu(u)-\int_{X} f \frac{d \mu^{y^{\prime}}}{d \nu} d \nu(u)\right| \\
& =\left|\int_{X} f\left(\frac{d \mu^{y}}{d \nu}-\frac{d \mu^{y^{\prime}}}{d \nu}\right) d \nu(u)\right| \\
& \leq \|\left. f\right|_{L_{\nu}^{\infty}} \cdot \int_{X}\left|\frac{d \mu^{y}}{d \nu}-\frac{d \mu^{y^{\prime}}}{d \nu}\right| d \nu(u) \\
& =\max \left(\|f\|_{L_{\mu^{y}}^{\infty}},\|f\|_{L_{\mu y^{\prime}}^{\infty}}\right) \cdot d_{T V}\left(\mu^{y}, \mu^{y^{\prime}}\right) .
\end{aligned}
$$

The second conclusion is a direct corollary from Thm. $22\left(d_{\text {Hell }}\left(\mu^{y}, \mu^{y^{\prime}}\right) \lesssim_{r}\left\|y-y^{\prime}\right\|_{\mathcal{Y}}\right)$ and Lem. $19\left(d_{T V}\left(\mu^{y}, \mu^{y^{\prime}}\right) \leq \sqrt{2} d_{H e l l}\left(\mu^{y}, \mu^{y^{\prime}}\right)\right)$.

\section{C.4 Measure preserving dynamics}

The following algorithm from [Dashti and Stuart, 2017] is proposed to sample distributions in the form of $\mu(d u)=\mu_{0}(d u) \frac{1}{Z} \exp (-\Phi)$ :

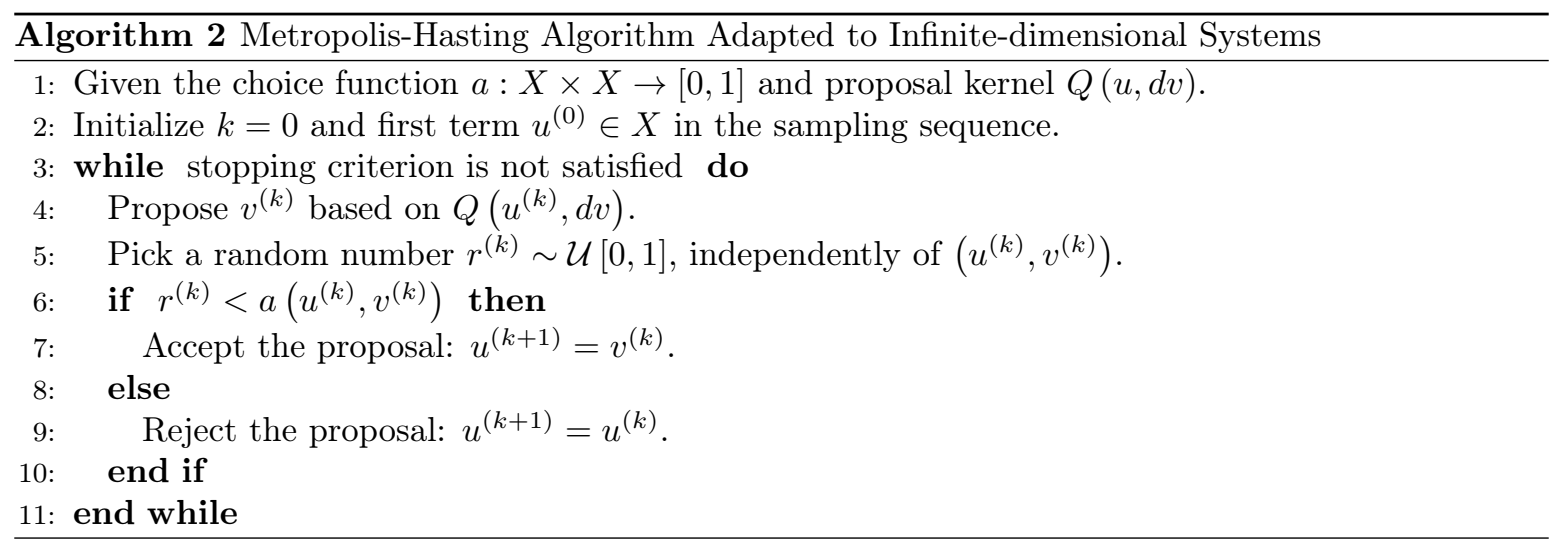

To preserve the desired measure, we can choose the choice function $a$ and proposal kernel $Q$ according to the following proposition.

Proposition 24. The Markov chain sampled in Alg. 2 is reversible to the measure $\mu \sim \mu_{0} \exp (-\Phi)$ if the following assumption holds: the negative $\log$ potential $\Phi: X \rightarrow \mathbb{R}$ is upper-bounded on any bounded set of $X$, the choice function $a(u, v)=\min \{1, \exp (\Phi(u)-\Phi(v))\}$ and the proposal kernel satisfies the reversible condition

$$
\mu_{0}(d u) Q(u, d v)=\mu_{0}(d v) Q(v, d u) .
$$

Note. The proof is on Page 53 in [Dashti and Stuart, 2017. 